\title{
The Assembly of Mammalian SWI/SNF Chromatin Remodeling Complexes is Regulated by Lysine- Methylation Dependent Proteolysis
}

Hui Zhang ( $\nabla$ hui.zhang@unlv.edu )

University of Nevada, Las Vegas https://orcid.org/0000-0001-6028-2554

Pengfei Guo

University of Nevada, Las Vegas

Nam Hoang

University of Nevada, Las Vegas

Joseph Sanchez

University of Nevada, Las Vegas

Elaine Zhang

University of California, Berkeley

Keshari Rajawasam

University of Nevada, Las Vegas

Kristiana Trinidad

University of Nevada, Las Vegas

Hong Sun

University of Nevada, Las Vegas

\section{Article}

Keywords:

Posted Date: January 18th, 2022

DOI: https://doi.org/10.21203/rs.3.rs-1139454/v1

License: (c) (1) This work is licensed under a Creative Commons Attribution 4.0 International License.

Read Full License

Version of Record: A version of this preprint was published at Nature Communications on November 5th, 2022. See the published version at https://doi.org/10.1038/s41467-022-34348-9. 


\section{Abstract}

The assembly of SWI/SNF chromatin remodeling complexes is developmentally programed, and loss/mutations of SWI/SNF subunits alter the levels of other components through proteolysis, causing cancers. We found that mouse $L s d 1 / K d m 1$ a deletion causes dramatic dissolution of SWI/SNF complexes and that LSD1 demethylates the methylated lysine residues in SMARCC1 and SMARCC2 to preserve the structural integrity of SWI/SNF complexes. The methylated SMARCC1/SMARCC2 are targeted for proteolysis by L3MBTL3 and the CRL4 ${ }^{\mathrm{DCAF5}}$ ubiquitin ligase complex. We identify SMARCC1 as the critical target of LSD1 and L3MBTL3 to maintain the pluripotency and self-renewal of embryonic stem cells. L3MBTL3 also regulates SMARCC1/SMARCC2 proteolysis induced by the loss of SWI/SNF subunits. Consistently, mouse $L 3 m b t / 3$ deletion causes striking accumulation of SWI/SNF components, associated with embryonic lethality. Our studies reveal that the assembly/disassembly of SWI/SNF complexes is dynamically controlled by a novel lysine-methylation dependent proteolytic mechanism to maintain the integrity of the SWI/SNF complexes.

\section{Introduction}

The mammalian SWI/SNF (mSWI/SNF, also called BRG1/BRM-Associated Factor, BAF) complexes are large ATP-dependent chromatin remodeling complexes assembled from up to 15 subunits with multiple paralogues during development to regulate stem cells, differentiation, and cell fate determination ${ }^{1,2}$. The developmentally programed $\mathrm{mSWI} / \mathrm{SNF}$ complexes assemble into cell/tissue-specific remodeling complexes by changing subunits or incorporating various subunit paralogues for the development of different tissues ${ }^{3}$. In mouse embryonic stem cells (mESCs) and pluripotent mouse F9 embryonic carcinoma cells, a unique SWI/SNF complex, the esBAF complex, is assembled with limited key subunits including BRG1 (SMARCA4 or BAF190A), the ATP-dependent chromatin remodeling catalytic subunit, SMARCC1 (BAF155 or SRG3), ARID1A (SMARCF1 or BAF250A), PBRM1 (Polybromo-1 or BAF180), and SMARCB1 (BAF47, INI1, or SNF5), to functionally associate with OCT4 and NANOG to regulate the central transcription circuitry of $m E S C s^{4-7}$. Loss of either BRG1 or SMARCC1 impairs the pluripotency and selfrenewal of mESCs ${ }^{4,5,7,8}$, whereas ectopic expression of BRG1 or SMARCC1 in mouse embryonic fibroblasts (MEFs) significantly enhances SOX2, OCT4, and Myc-mediated reprogramming into induced pluripotent stem cells (iPSCs) ${ }^{8}$. When mESCs undergo differentiation, additional mSWI/SNF proteins, such as BRM (SMARCA2 or BAF190B), a BRG1 paralogue, and SMARCC2 (BAF170), a SMARCC1 paralogue, are co-expressed to form BRG1- and BRM-based SWI/SNF complexes ${ }^{6}$. In the mouse, homozygous deletions of $B r g 1$ or Smarcc 1 are early peri-implantation lethal during embryogenesis ${ }^{9,10}$, whereas Brm is dispensable and Smarcc2 null mutant animals die shortly after birth ${ }^{11}$. Recent human cancer exome and whole-genome sequencing studies revealed that loss or mutations of mSWI/SNF genes encoding various subunits are associated with more than $20 \%$ of human cancers ${ }^{2,3}$. The mutations or loss of a single mSWI/SNF subunit gene often uniquely cause the proteolysis of other subunits to assemble aberrant mSWI/SNF complexes with changes in stoichiometry and composition ${ }^{12-15}$. Notably, cancer cells defective in a particular m/SNF subunit are often vulnerable to synthetic lethality of losing its 
remaining paralogue in the residual SWI/SNF complexes ${ }^{13-17}$. For example, cancer cells defective in BRG1 are usually sensitive to the removal of BRM, whereas cancer cells with ARID1A mutations are vulnerable to the loss of ARID1B ${ }^{13-17}$. However, little is known about how the assembly or disassembly of $\mathrm{mSWI} / \mathrm{SNF}$ complexes and the proteolysis of subunits are regulated during development and tumorigenesis.

Lysine methylation is an important post-translational protein modification and lysine residues in proteins can be mono-, di-, and tri-methylated. Extensive research has established the key roles of various methylated lysine residues at the amino terminal regions of histones in modulating chromatin structure and gene expression ${ }^{18,19}$. While trimethylations at lysines 9 and 27 in histone $\mathrm{H} 3$ (H3K9me3 and H3K27me3) are usually associated with repressive chromatin structure that suppresses gene expression, the trimethylation at lysine 4 in histone H3 (H3K4me3) typically associates with active transcription ${ }^{18}$. LSD1 (also called KDM1a) was originally identified as a histone demethylase that specifically removes methyl groups from the mono- and dimethylated lysine 4 in histone $\mathrm{H} 3$ (H3K4me1/me2), but not trimethylated H3K4, to repress transcription ${ }^{18,20}$. Null deletion of mouse Lsd1 gene causes early embryonic lethality around embryonic day $5.5(E 5.5)^{21,22}$. Loss of LSD1 also profoundly impairs the pluripotency and self-renewal of embryonic stem cells (ESCs) and the differentiation of other stem/progenitor cells such as mouse hematopoietic stem cells ${ }^{23-26}$. The tamoxifen-induced conditional null deletion of the floxed $L s d 1$ mice (4-9 weeks old) by actin-CreER (CAGGCre-ER) in the hippocampal and cerebral cortex neurons leads to paralysis with widespread hippocampus and cortex neurodegeneration, as well as learning and memory defects ${ }^{27}$. However, the molecular targets of LSD1 deficiency that cause the devastating animal phenotypes remains unclear.

Many non-histone proteins, such as p53, DNA (cytosine-5)-methyltransferase 1 (DNMT1), SOX2, LIN28A, HIF1a, NFkB/RelA, ERa, GLI3, and E2F1, are mono-methylated on specific lysine residues by SET7 (SET7/9, SET9, SETD7 or KMT7) ${ }^{25,26,28-32}$, which was originally isolated as a histone methyltransferase that mono-methylates $\mathrm{H} 3 \mathrm{~K} 4^{33,34}$. Accumulating evidence indicates that methylation of lysine residues by SET7 on a group of non-histone proteins, such as DNMT1, E2F1, SOX2, NFkB/RelA, FOXO3, and STAT3 ${ }^{35-}$ 40 , triggers the proteolytic destruction of these modified proteins. We and others have recently shown that LSD1 acts as a demethylase to remove the methyl groups of mono-methylated lysine residues in DNMT1, E2F1, and SOX2 proteins to prevent these proteins from the methylation-dependent proteolysis $25,26,37,41$. Here, we found that LSD1 also acts as a demethylase to regulate the assembly of mSWI/SNF complexes by preventing the proteolysis of $\mathrm{mSWI} / \mathrm{SNF}$ subunits through a lysine-methylation dependent proteolytic mechanism.

\section{Results}


To understand the molecular mechanism by which $L s d 1$ null deletion causes embryonic lethality and other devastating effects in animals, we bred the floxed $L s d 1$ conditional deletion mouse strain ${ }^{24}$ with the nestin-Cre transgenic mice ${ }^{42}$ to specifically delete $L s d 1$ in the neuronal and glial cell precursors of the central nervous system. We found that the nestin-Cre-mediated homozygous loss of $L s d 1$ conditional alleles caused post-natal lethality immediately after birth (P0, Fig. 1a). During the characterization of $L s d 1$ mutants, we repeatedly observed that the protein levels of several subunits of mSWI/SNF chromatin remodeling complexes, such as BRG1, SMARCC1, SMARCC2, ARID1A, and PBRM1, are dramatically reduced in the brain extracts of LSD1 null mutants, as compared with that of wildtype littermates (Figure 1b). Consistently, our immunostaining of brain sections from the wildtype and $L s d 1$ null mutant animals also revealed that the protein level of SMARCC1, a core mSWI/SNF component ${ }^{14,43-45}$, is markedly reduced in the $L s d 1$ mutant mice (Fig.1c). To rule out potential degradation of mSWI/SNF proteins caused by animal lethality, we bred $L s d 1$ conditional deletion mouse strain ${ }^{24}$ with a transgenic mouse line expressing a tamoxifen inducible Cre-ER recombinase under the Actin promoter control (CAGGCre$E R)^{46}$ to establish mouse embryonic fibroblasts (MEFs) from the homozygous floxed LSD1 conditional $(\mathrm{fl} / \mathrm{fl})$ deletion mouse embryos with the actin-Cre-ER (CAGGCre-ER/Lsd1fl/fl). While these MEFs normally express significant levels of $\mathrm{mSWI} / \mathrm{SNF}$ proteins, induced deletion of $L s d 1$ in the CAGGCre-ER/Lsd17l/fl MEFs by addition of 4-hydroytamoxifen (4-OH-Tam) led to the rapid disappearance of these mSWI/SNF proteins (Fig. 1d). Our studies indicate that LSD1 is required to maintain the protein stability and integrity of $\mathrm{mSWI} / \mathrm{SNF}$ complexes.

\section{SMARCC1 and SMARCC2 are targets of LSD1 and L3MBTL3}

Mutations or loss of a single critical mSWI/SNF subunit, such as SMARCB1 in malignant rhabdoid tumors and epithilioid sarcoma, ARID1A in a wide variety of cancers including ovarian clear cell carcinoma, endometrioid carcinoma, neuroblastoma, and bladder cancer, BRG1 in medulloblastoma, breast and lung caners, SMARCC1 in small cell lung cancer, and SMARCC2 in pancreatic cancer, uniquely causes the proteolysis of other subunits ${ }^{2,3,12-15}$. Recent studies indicate that the structural integrity of the mSWI/SNF complexes requires the formation of homo- or heterodimer of SMARCC1 and SMARCC2 ${ }^{43}$ and loss of SMARCC1 and/or SMARCC2 causes the disassembly of mSWI/SNF complexes and proteolytic degradation of many mSWI/SNF subunits ${ }^{15,44,45}$. As LSD1 serves as a demethylase for DNMT1, E2F1, and SOX $2^{25,26,41}$, we wondered whether $\mathrm{mSWI} / \mathrm{SNF}$ proteins are regulated by lysine methylation. While lysine methylations of $\mathrm{mSWI} / \mathrm{SNF}$ proteins have not been reported, our protein sequence examination revealed that SMARCC1 and SMARCC2 both contain several H3K4-like lysine residues that may be monomethylated by the SET7 methyltransferase (Fig. 3a). To test whether SMARCC1 or SMARCC2 is regulated by SET7, we used siRNA-mediated silencing of SET7 and found that loss of SET7 indeed increased the levels of both SMARCC1 and SMARCC2 proteins (Fig. 1e). While LSD1 siRNAs reduced the protein levels of SMARCC1 and SMARCC2, co-silencing of SET7 and LSD1 effectively re-stabilized SMARCC1 and SMARCC2 proteins in LSD1 deficient cells (Fig. 1e). We also stably expressed Flag-tagged SMARCC1 or 
SMARCC2 under the retroviral LTR promoter control ${ }^{41}$ in $\mathrm{H} 1299$ cells and found that the ectopically expressed Flag-SMARCC1 and SMARCC2 are also sensitive to LSD1 silencing (Fig. 1f, Fig. $4 \mathrm{~g}$ and 4h). LSD1-silencing induced Flag-SMARCC1 downregulation is also blocked by 26S proteasome inhibitor, MG132 (Fig. 1f) ${ }^{25,26}$. These observations indicate that loss of LSD1 induces the proteolysis of SMARCC1 and SMARCC2 proteins through the ubiquitin-dependent pathway.

\section{L3MBTL3 regulates the proteolysis of SMARCC1 and SMARCC2}

Since LSD1 is a demethylase, it is possible that SMARCC1 and SMARCC2 might be lysine methylated to trigger their proteolysis. We have recently shown that the mono-methylated lysine residues in DNMT1, E2F1, and SOX2 are recognized by L3MBTL3, a Malignant Brain Tumor (MBT) domain protein that specifically binds to methyl-lysine residues ${ }^{47}$, to target substrate proteolysis by the CRL4 ${ }^{\mathrm{DCAF} 5}$ ubiquitin E3 ligase complex ${ }^{26,41}$. We wondered whether L3MBTL3 and DCAF5, a substrate-specific subunit of CRL4 that interacts with $L 3 M B T L 3^{41}$, regulate the proteolysis of SMARCC1 or SMARCC2 in LSD1 silenced cells. Indeed, siRNA-mediated silencing of L3MBTL3 stabilized both SMARCC1 and SMARCC2 proteins in LSD1 deficient cells (Fig. 2a and 2b). Furthermore, while LSD1 silencing led to the downregulation of other $\mathrm{mSWI} / \mathrm{SNF}$ proteins such as BRG1, BRM, ARID1A, and PBRM1, co-silencing of L3MBTL3 and LSD1 reversed these downregulations in LSD1 silenced cells (Figure 2c). Furthermore, our immuno-coprecipitation analysis revealed that endogenous L3MBTL3 and SMARCC1 proteins reciprocally interact with each other (Fig. 2f), and this interaction is significantly enhanced in the presence of SET7 (Fig. 2g). In addition, while LSD1 silencing induces the degradation of ectopically expressed Flag-SMARCC1 protein, such degradation is prevented by co-silencing of L3MBTL3 or DCAF5 and LSD1 (Fig. 2d and 2e). Moreover, either the presence of SET7 or LSD1 silencing can stimulate SMARCC1 polyubiquitination, which is further promoted by co-expression of SET7, L3MBTL3, and DCAF5 (Fig. 2h and supplementary Fig. 1c).

SMARCC1 contains three lysine residues, Lys201 (K201), Lys482 (K482), and Lys615 (K615) in the putative H3K4-like methylation consensus motifs (Fig. 3a) ${ }^{25,26,28,41}$ that are conserved (K482) or partially conserved (K615) in the K475 or the K592 motif of SMARCC2, respectively. To test whether these putative methylated lysine residues in SMARCC1 serve as substrates for L3MBTL3 and LSD1, we synthesized mono-methylated K482 and K615 peptides and their unmethylated cognate peptides derived from SMARCC1 (Fig. 3b). We found that in vitro translated and ${ }^{35}$ S-labeled L3MBTL3 protein selectively and directly binds to the methylated K482 and K615 peptides immobilized onto Sepharose beads ${ }^{41}$, but not to their cognate unmethylated peptide beads (Figure 3b). We also examined whether LSD1 can remove the methyl groups from the synthetic mono-methylated K482 and K615 peptides by mixing the methylated peptides with the purified recombinant GST-LSD1 fusion protein or GST control ${ }^{25,41}$. We found that GST- 
LSD1 can effectively demethylate the methylated K482 or K615 peptides (Figure 3c and 3d, and Supplementary Fig. 1a and 1b).

To further define the critical lysine residues in SMARCC1, we mutated K201, K482, or K615 of SMARCC1 to arginine to produce $\mathrm{K} 201 \mathrm{R}, \mathrm{K} 482 \mathrm{R}$, and K615R mutants either singly or in combination so that they cannot be methylated by SET7 (Fig. 3a). Expression of K201R/K482R or K201R/K615R double mutants indicate that the presence of either K615 or K482 is sufficient for SMARCC1 to interact with L3MBTL3 (Fig. 4a and 4b). However, the K201R/K482R/K615R triple mutant (TM) of SMARCC1 failed to interact with L3MBTL3 (Fig. 4c). Consistently, while the K201R/K482R and K201R/K615R double mutants of SMARCC1 are still sensitive to LSD1 silencing, the K482R/K615R double mutant and in particular the K201R/K482R/K615R triple mutant are much more resistant to LSD1 silencing (Figure 4d and 4e). Furthermore, the K201R/K482R/K615R triple mutant is also resistant to be polyubiquitinated by SET7, L3MBTL3, and DCAF5 (Fig. 2h). Our results indicate that the methylated SMARCC1 protein is directly recognized by L3MBTL3 to promote its ubiquitin-dependent degradation by the CRL4 ${ }^{\text {DCAF5 }}$ ubiquitin E3 ligase and that LSD1 acts as a demethylase to remove the methyl groups from methyl-lysine 482 and methyl-lysine 615 of SMARCC1 to prevent the proteolysis of SMARCC1 by L3MBTL3 and the CRL4 DCAF5 ubiquitin E3 ligase complex.

Since SMARCC2 also contains lysine residues in the putative H3K4-like methylation motifs (Fig. 3a), our studies revealed that the binding of SMARCC2 to L3MBTL3 is stimulated by SET7 (Fig. 4f). We also constructed the lysine-to-arginine mutations of either K475 alone, which is identical to K482 in SMARCC1, or triple mutations of K382/K475/K592 of SMARCC2 (Fig. 3a). We found that the K475R single and the K382R/K475R/K592R triple mutants (TM) of SMARCC2 are much more resistant to the loss of LSD1 (Fig. $4 \mathrm{~g}$ and $4 \mathrm{~h}$ ). Our studies indicate that LSD1 and L3MBTL3 act through the H3K4-like lysine residues, such as K475 of SMARCC2 to regulate SMARCC2 protein stability.

\section{Regulation of SMARCC1 and mSWI/SNF complexes in mouse embryonic stem cells}

Mouse embryonic stem cells (mESCs) and mouse F9 embryonic carcinoma cells uniquely express a specific mSWI/SNF complex, the esBAF complex, that only contains SMARCC1 but not SMARCC2 ${ }^{4-7}$ (Supplementary Fig. 2c). We found that siRNA-mediated silencing of LSD1 caused the proteolysis of SMARCC1 and greatly impaired the self-renewal and pluripotency of mESCs, indicated by the loss of alkaline phosphatase (AP) activity (Fig. 5a and 5b). SMACC1 degradation can be reversed by L3MBTL3 silencing in LSD1 deficient mESCs (Fig. 5a), indicating that L3MBTL3 mediates the proteolysis of SMARCC1 in mESCs ${ }^{26}$. LSD1 silencing also blocked the proliferation of F9 cells and induced SMARCC1 degradation, and reduction of mSWI/SNF proteins (Fig. 6a, Supplementary Fig. 1d and 2a-2c). The 
effects of LSD1 silencing are unique for mESCs and F9 cells, as silencing of LSD1 in human embryonic kidney carcinoma 293 and lung carcinoma H1299 cells that express both SMARCC1 and SMARCC2 did not exhibit any proliferation defects (Supplementary Fig. 2a-2c). While our studies and other reports indicate that LSD1 plays an essential role in both human and mouse ESCs $22,23,25,26$, the mechanism by which LSD1 regulates ESCs remains unclear. As SMARCC1 silencing impairs the self-renewal of both mESCs and F9 cells but not the proliferation of 293 or H1299 cells ${ }^{4,5}$ (Supplementary Fig. 2a and 2b), similar to the selective effects of LSD1 silencing in these cells, we ectopically and stably expressed FlagSMARCC1 and its lysine-to-arginine mutants using the PMSCV retroviral LTR vector in both $\mathrm{mESC}$ and F9 cells. Strikingly, we found that stable expression of the K201R/K482R/K615R triple mutant of FlagSMARCC1, but not the wildtype counterpart, are sufficient to confer the resistance to LSD1 silencing to maintain the pluripotency and self-renewal in mESCs (Fig. 5b and 5c). Similarly, expression of FlagSMARCC1 triple mutant, as well as the K482R/K615R double mutant, in F9 cells are sufficient to render the resistance to LSD1 silencing (Fig. 6a, 6b, 6d, and 6e). The effect of LSD1 silencing appears to resemble the loss of SMARCC1, as loss of either LSD1 or SMARCC1 blocked cell proliferation, induced the disassembly of the mSWI/SNF complex, and the degradation of mSWI/SNF subunits, as analyzed by the immuno-co-precipitation of the mSWI/SNF complex by anti-SMARCB1 antibodies (Fig. 6b, 6c, and Supplementary Fig. 1d). However, expression of the Flag-SMARCC1 triple mutant is sufficient to confer the resistance to LSD1 deficiency-induced growth inhibition, disassembly of the mSWI/SNF complex, and subunit degradation (Fig. 6b and 6c, and Supplementary Fig. 1d). These studies suggest that SMARCC1 is a key target of LSD1 in both mESCs and F9 cells.

The mSWI/SNF complex is essential for mESCs and F9 cells through their regulation of the central OCT4SOX2-NANOG transcription circuitry ${ }^{4-7}$. Indeed, silencing of SMARCC1 caused the downregulation of OCT4 and SOX2, and the upregulation of NANOG in mESCs (Fig. 5d). We have previously shown that loss of LSD1 causes the downregulation of SOX $2^{25,26}$. Notably, while LSD1 silencing reduced SOX2 levels in mESCs or F9 cells expressing the wildtype Flag-SMARCC1, expression of the K482R/K615R double or the triple mutant or mutant of Flag-SMARCC1 is sufficient to reverse the downregulation of SOX2 after LSD1 silencing in mESCs and F9 cells (Fig. $5 \mathrm{c}$ and $6 \mathrm{e}$ ). We also found that SMARCC1 and SOX2 interact with each other in mESCs. These studies suggest that SMARCC1 is a critical target of LSD1 in regulating the assembly of mSWI?SNF complex in MESCs and F9 cells and that there is a direct link between the integrity of $\mathrm{mSWI} / \mathrm{SNF}$ complex and SOX2 to maintain the self-renewal and pluripotency of mESCs and F9 cells.

\section{Cell cycle regulation of mSWI/SNF proteins by L3MBTL3}

We repeatedly found that aphidicolin, a DNA polymerase inhibitor that arrests replicating cells at the G1/S border and in S phase, reduced the steady state levels of SMARCC1 and BRG1 in mESCs or HeLa cells, as 
compared to asynchronously growing cells (Fig. 5f, 5g, and Supplementary Fig. 2d). The aphidicolinarrested cells are associated reduced levels of SOX2 and increased levels of cyclin E (Fig. $5 \mathrm{~g}$ ), a late G1 and early $S$ phase marker, in mESCs ${ }^{41}$. Notably, in aphidicolin-arrested S phase mESCs, L3MBTL3 silencing is sufficient to restore the levels of SMARCC1, BRG1, and SOX2 (Fig. 5g). To characterize potential cell cycle regulation of mSWI/SNF complexes, we synchronized HeLa cells at the G1/S bounder by the sequential thymidine/aphidicolin treatment ${ }^{41}$, and measured the levels of mSWI/SNF proteins after releasing synchronized cells into $S$ phase in fresh media without aphidicolin. Our studies revealed that the protein levels of SMARCC1, SMARCC2, BRG1, PBRM1, and ARID1A are relatively low in the synchronized $\mathrm{G} 1 / \mathrm{S}$ and early $\mathrm{S}$ phase cells, and their levels become gradually increased as cells progress towards late S phase and/or G2 phase (Supplementary Fig. 2e). We could also detect the K615methylated SMARCC1 in these synchronized cells (our anti-methylated K482 antibody has high background). These observations are consistent with our previous findings that L3MBTL3-regulated DNMT1 degradation increases in S phase and that SOX2 is a substrate of L3MBTL3 ${ }^{25,26,41}$. Our findings are also consistent with previous observations that SMARCC2 protein is undetectable in S and G2 phases of proliferating embryonic cortical progenitor cells during mouse cortex development between E10.5$\mathrm{E} 14.5^{15}$. Together, these observations suggest that the levels of $\mathrm{mSWF} / \mathrm{SNF}$ proteins are cell cycle regulated by L3MBTL3.

\section{L3MBTL3 regulates the proteolysis of SMARCC1 and SMARCC2 induced by mSWI/SNF subunit loss}

It is well established that the subunit stoichiometry and composition of mSWI/SNF complexes are uniquely regulated that mutation/loss of a particular subunit, such as SMARCC1, SMARCC2, SMARCB1, BRG1, or ARID1A, destabilizes the mSWI/SNF complexes to induce proteolysis of other subunits via an unknown mechanism (Fig. 7a) ${ }^{14,44,45,48}$. Indeed, our studies showed that silencing of either SMARCC2 or SMARCB1 triggered the proteolysis of mSMARCC1 and other mSWF/SNF proteins such as PBRM1 (Fig. 6b, 6c, 7b, and 7c). We wondered whether L3MBTL3 and CRL4 are involved in SMARCC1 degradation during the $\mathrm{mSWI}$ SNF disassembly induced by the loss of SMARCC2 or SMARCB1. Our tests revealed that SMARCC1 degradation, induced by SMARCC2 silencing, can be effectively reversed by MLN4924, a protein neddylation inhibitor that blocks the assembly of CRL ubiquitin ligase complexes including CRL4 ${ }^{49}$ (Fig. 7b). Importantly, we repeatedly found that while silencing of SMARCC2 or SMARCB1 triggers the degradation of the wildtype Flag-SMARCC1, the Flag-SMARCC1 triple mutant is resistant to the loss of either SMARCC2 or SMARCB1 (Fig. 7c, 7e and Supplementary Fig. 1e), and that the triple SMARCC1 mutant expression also stabilized other mSWI/SNF proteins such as PBRM1. If SMARCC1 proteolysis during the silencing of SMARCC2 is mediated by L3MBTL3, we would expect that the interaction between SMARCC1 and L3MBTL3 would increase. Indeed, we found that SMARCC2 silencing enhanced the binding of SMARCC1 to L3MBTL3, suggesting that the released SMARCC1 after the disassembly of mSWI/SNF complexes may facilitate SMARCC1 methylation and its subsequent binding to L3MBTL3 (Fig. 7a and 7d). Conversely, while SMARCC1 silencing induces proteolysis of the wildtype Flag- 
SMARCC $^{14,44,45}$, the K475R and the triple mutants of Flag-SMARCC2 are more resistant to SMARCC1 silencing (Fig. 7f). Thus, our results reveal that the proteolysis of SMARCC1 or SMARCC2 triggered by the loss of individual subunit of mSWI/SNF complexes is regulated by the L3MBTL3-CRL4 ${ }^{\text {DCAF5 }}$ dependent pathways.

\section{Regulation of SMARCC1 and SMARCC2 by L3MBTL3 during embryonic development}

Human $\angle 3 M B T L 3$ is mutated in medulloblastoma and is further implicated in other pathological disorders such as multiple sclerosis, insulin resistance, prostate cancer and breast cancer ${ }^{47,50-53}$. Mouse homozygous $L 3 m b t / 3$ deletion is late embryonic lethal around E17.541,54, although the molecular targets for the lethality remain unclear (Fig. 8a). We found that the protein levels of mSWI/SNF subunits including SMARCC1, BRG1, SMARCC2, PBRM1, ARID1A, and SMARCB1 are markedly elevated in the head extracts of mouse $\angle 3 m b t /(-/-)$ deletion mutant embryos at E14.5, as compared to that of littermates (Fig. $8 b)$. Further characterization showed that the protein levels SMACC1 were high and comparable in both wildtype and $\angle 3 m b t / 3$ deletion mutant embryos at E9.5. However, while in wildtype embryos, SMARCC1 protein levels became gradually declined between E14.5-E17.5, the protein levels of SMARCC1 accumulated in the $\angle 3 m b t / 3$ deletion embryos in this developmental period and these $L 3 m b t / 3$ deficient embryos eventually died ${ }^{41}$ (Fig. 8a and 8c). These studies indicate that SMARCC1 protein is targeted for proteolysis by L3MBTL3 between E14.5-E17.5. In MEFs isolated from L3mbt/3 homozygous deletion mutant embryos (Figure $8 \mathrm{~d}$ ), mSWI/SNF proteins also accumulated and $\mathrm{L} 3 \mathrm{mbt} / 3$ deletion markedly increased the methylated K615 level in SMARCC1, as compared to the MEFs from the wildtype littermates (Fig. 8e). Immunostaining of brain tissues revealed that the protein levels of total and K615-methylated SMARCC 1 and SMARCC2 are significantly elevated in $\angle 3 m b t / 3$ deleted embryos, as compared to that of wildtype littermates (Fig. 8f). These studies indicate that L3MBTL3 regulates the proteolysis of SMARCC1 and SMARCC2 during embryonic brain development and that the accumulation of mSWI/SNF proteins may contribute to the embryonic lethality of $L 3 m b t 3$ deletion mutants. Our studies are consistent with a model by which SMARCC1 and SMARCC2 are methylated by SET7 to recruit L3MBTL3 and its associated CRL4 ${ }^{\text {DCAF5 }}$ ubiquitin ligase complex to target SMARCC1 and SMARCC2 proteins for degradation, thereby regulating the disassembly of the $\mathrm{mSWI} / \mathrm{SNF}$ complex through the subsequent proteolysis of other $\mathrm{mSWI} / \mathrm{SNF}$ subunits, and that LSD1 serves as a demethylase to remove the methyl groups from the methylated SMARCC1 and SMARCC2 proteins to prevent their degradation to preserve the integrity of the mSWI/SNF complexes (Fig. 8g).

\section{Discussion}

In this report, we found that the assembly or disassembly of the mSWI/SNF chromatin remodeling complexes are dynamically regulated by a novel lysine methylation dependent proteolytic mechanism involving the LSD1 demethylase and L3MBTL3-CRL4 ${ }^{\text {DCAF5 }}$ ubiquitin ligase activities. Our studies revealed that two key mSWI/SNF components, SMARCC1 and SMARCC2, contain critical lysine residues for 
methylation and these methylated lysine residues serve as the substrates of LSD1 and L3MBTL3 that regulate the proteolysis of these two proteins, promoting the disassembly of the mSWI/SNF complexes. Our studies are consistent with previous findings that SMARCC1 and SMARCC2 are the core mSWI/SNF components that are present in every mSWI/SNF complex for the nucleation of the mSWI/SNF complex assembly and that loss of SMARCC1 and SMARCC2 leads to the disassembly of entire mSWI/SNF complexes and the degradation of many mSWI/SNF subunits ${ }^{15,43-45}$. Notably, loss of LSD1 blocked the self-renewal of mESCs and F9 cells that express the ESC-specific esBAF complex that contains only SMARCC 1 1,5, and our work identifies SMARCC1 as the critical target of LSD1 in mESCs and F9 cells (Fig. 5 and 6). However, loss of LSD1 in other cells, such as 293 or H1299 cells that contain both SMARCC1 and SMARCC2, has no detectable growth defects (Supplementary Fig. 2a and 2b), although the loss of LSD1 triggers the degradation of both SMARCC1 and SMARCC2 in these cells. It is possible that the fractions of the methylated SMARCC1 and/or SMARCC2 proteins are not high enough to cover every mSWI/SNF complex in 293 and H1299 cells. SMARCC1 or SMARCC2 are also proteolyzed when mSWI/SNF subunits are lost or mutated ${ }^{14,44,45,48}$. Our evidence suggests that the proteolysis of SMARCC1 and SMARCC2 during the loss of mSWI/SNF subunits is L3MBTL3 dependent (Fig. 7). Although we found that SMARCC1 and SMARCC2 are regulated through lysine methylation, it remains to be further investigated whether additional $\mathrm{mSWI}$ /SNF proteins are similarly regulated by lysine methylation dependent proteolysis through LSD1 and the L3MBTL3-CRL4 ${ }^{\text {DCAF5 }}$ ubiquitin ligase complexes.

\section{Methods}

Cells: Human lung carcinoma H1299, cervical carcinoma HeLa, embryonic kidney 293, and mouse teratoma F9 cells were purchased from the American Type Culture Collection (ATCC) and cultured in RPMI-1640 or DMEM medium with $10 \%$ FBS and $1 \%$ antibiotics as described ${ }^{25,26,41}$. Mouse embryonic fibroblasts (MEFs) were generated from wildtype and $\angle 3 m b t / 3$ deletion mutant embryos or CAGGCre-

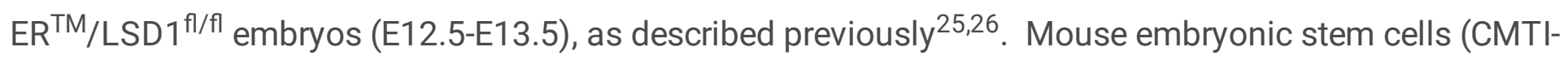
2, strain C57/ BL6J, passage 16) were obtained from Millipore-Sigma and were cultured on the mitomycin $\mathrm{C}$ treated mouse fibroblast feeder layer in knockout DMEM and knockout serum replacement, supplemented with leukemia inhibitory factor (LIF), GlutaMax, $\beta$-mercaptoethanol, MEM nonessential amino acid solution, and antibiotics ${ }^{25,26}$ (all from Life Technologies). For stable expression, human SMARCC1 and SMARCC2 were cloned into the retroviral pMSCV-Puro vector containing 3xFlag-3xHA epitope (clontech) and the recombinant retroviruses were packaged in 293 cells $^{25,26}$. Viral infected H1299, F9 and mouse ES cells were selected by puromycin resistance. For cell cycle synchronization, actively growing Hela cells were treated with $2.5 \mathrm{mM}$ thymidine for 18 hours, released into fresh cell culture medium for 9 hours, and then treated again with $5 \mu \mathrm{g} / \mathrm{ml}$ aphidicolin (Sigma) for another 15 hours to synchronously arrest the cells at the G1/S border ${ }^{41}$. 


\section{Peptide synthesis and preparation of methylated peptides:}

The monomethylated K482 (RALPEFFNGKNKS ${ }_{(\mathrm{Km} 1)}$ TPEIYLAYRNFMIDTC) and K615 (GLRTDIYSKKTLAKS $_{(\mathrm{Kme} 1)}$ GASAGREWTEQETC) and cognate unmethylated peptides of SMARCC1 were synthesized at ABI Scientific. The monomethylated K482 and K615 peptides were used to raise rabbit polyclonal antibodies after coupling these peptides to keyhole limpet hemocyanin (KLH) ${ }^{41}$. Affinity purification of methylated peptide antibodies were conducted as described ${ }^{41}$, the unmethylated and monomethylated K482 or K615 peptides were immobilized to Sulfolink-coupled-resins (Thermo Fisher) by covalently cross-linking with the cysteine residues at the end of the peptides to the resin ${ }^{41}$. The antimonomethylated $\mathrm{K} 482$ or $\mathrm{K} 615$ peptide sera ( $5 \mathrm{ml}$ each) were diluted in 1:1 in PBS and first passed through the unmethylated $\mathrm{K} 482$ or unmethylated $\mathrm{K} 615$ peptide columns $(1 \mathrm{ml})$ for three times to deplete anti-K482 or K615 peptide antibodies. The unbound flow-through antibody fractions were then loaded onto the monomethylated $\mathrm{K} 482$ or monomethylated $\mathrm{K} 615$ peptide column $(0.5 \mathrm{ml})$, washed, and the bound antibody fractions were eluted by $5 \mathrm{ml}$ of $100 \mathrm{mM}$ glycine, $\mathrm{pH} 2.5$. The eluted antibodies $(0.5$ $\mathrm{ml} /$ fraction) were immediately neutralized by adding $100 \mathrm{ml}$ of $2 \mathrm{M}$ Tris, pH8.5, and tested for specificity towards the monomethylated K482 or K615 peptide but not to the unmethylated K482 or K615 peptide. For L3MBTL3 binding to methylated peptides, the monomethylated K482 and K615 peptides and cognate unmethylated peptides of SMARCC1 were covalently coupled to the Sulfolink-coupled resins, respectively (Thermo Fisher) through the disulfide bond between the C-terminal cysteine of the peptides and the resins $^{25,26,41}$. Human L3MBTL3 was cloned into pcDNA3 and in vitro translated and isotope labeled using T7 polymerase TNT ${ }^{\circledR}$ Quick Coupled Transcription/Translation System (Promega) in the presence of ${ }^{35} \mathrm{~S}$ L-methionine (PerkinElm).

Antibodies and immunological analysis: Anti-LSD1 (A300-215A), L3MBTL3 (A302-852), SMARCC2 (A301038A), PBRM1 (A700-019), ARID1A (A301-040A), SMARCB1 (A301-087A), BRG1 (A301-087A), BRM (A301-015A), and SET7 (A301-747A) antibodies were purchased from Bethyl Laboratories; anti-BRG1 (49360) and anti-SMARCC1 (11956) were from Cell Signaling technology; anti-SMARCC1 (sc-32763) and actin (Sc-1616) antibodies were from Santa Cruz Biotechnologies. Anti-Flag, HA, and GFP antibodies were purchased from Sigma. Anti-L3MBTL3 and anti-DCAF5 antibodies were previously described. For direct western blotting, cells were lysed in the 1XSDS sample buffer (4\% SDS, $100 \mathrm{mM}$ Tris, pH6.8, and 20\% glycerol), quantified by protein assay dye (Bio-Rad), and equalized by total proteins ${ }^{41}$. For immunoprecipitation (IP), cells were lysed with a NP40-containing lysis buffer (0.5\% NP40, $50 \mathrm{mM}$ Tris, $\mathrm{pH} 7.5,150 \mathrm{mM} \mathrm{NaCl}$, and protease inhibitor cocktails $)^{41}$. About $500 \mu \mathrm{g}$ of lysates were used for each IP assay. The antigen-antibody complexes were then pulled down by $30 \mu \mathrm{l}$ Protein A-Sepharose (GE Healthcare) and specific proteins were detected by Western blotting analysis, using secondary goat antimouse HRP (Jackson Immuno Research, 115-035-008) and goat-anti-rabbit antibodies (Jackson Immuno Research, 111-035-008), or Protein A HRP (GE Healthcare, NA9120V), all at 1:5000 dilutions. 
Transfection and siRNAs: Oligofectamine was used for siRNA silencing in HeLa, H1299 or 293 cells, and DharmaFECT1 was used for the siRNA silencing in mouse ES and F9 cells, whereas Lipofectamine 2000 was used for transient transfection as described previously $25,26,41$. Typically, $50 \mathrm{nM}$ of each siRNA or their combinations were transfected into target cells for 48 hours and cells were directly lysed in SDS or NP40 lysis buffers ${ }^{41}$. For verification of the effects of new proteins, usually 2 or 3 independent siRNAs were designed to examine the knockdown efficiency and the consequences of knockdown on target proteins. The siRNAs for human genes are: LSD1: GGAAGAAGAUAGUGAAAAC; DCAF5:

GCUGCAGAAACCUCUACAA; L3MBTL3-1: GATGCAGATTCTCCTGATA; L3MBTL3-2: GGTACCAACTGCTC-

AAGAA; SMARCC1: GGAUGAAUGAGGAGGAUUAUU; SMARCC2: GCACA-

GACAUGUACACAAAUU; SMARCB1-1: ACACUAAGGAUCACGGAUAUU; SMARCB1-2:

ACGCUGAGAUGGAGAAGAAUU; SET7: GGGCAGTATAAAGA-

TAACA. The siRNAs for mouse genes are: mouse L3MBTL3: GCTGAGGTTTGTGGATATA; mouse L3MBTL3: GCTGAGGTTTGTGGATATA; mouse SMARCC1: GCUAACAAGUUGAAGAUAUU; mouse LSD1 is the same as human's, and control siRNA for luciferase: CATTCTATCCTCTAGAGGA. All siRNAs were synthesized from Horizon Discovery.

\section{Demethylation analysis}

The glutathione-S-transferase (GST) and the GST-LSD1 (human) fusion protein were expressed in E. coli BL21 strain and purified by the Glutathione Sepharose resin. Purified $1 \mathrm{mg}$ of control GST or GST-LSD1 proteins were incubated with $100 \mathrm{ng}$ of the monomethylated K482 or monomethylated K615 peptides for 4 hours at room temperature and the resulting peptides were blotted onto nitrocellulose membrane ${ }^{25,41}$. The demethylated peptides were detected by immuno-blotting with affinity purified anti-monomethylated K482 or anti-monomethylated K615 antibodies.

Animals and Histology. The LSD1fl/+ conditional mutant (B6.129-Kdm1a tm1.1Sho/J, stock No: 023969), transgenic actin-Cre-ER (CAGGCre-ER ${ }^{\mathrm{TM}}, \mathrm{B} 6 . \mathrm{Cg}-\mathrm{Tg}(\mathrm{CAG}$ cre/Esr1*)5Amc/J, stock No: 004682), and transgenic Nestin-Cre (B6.Cg- $\mathrm{Tg}(\mathrm{Nes}-\mathrm{cre}) 1 \mathrm{KIn} / \mathrm{J}$, stock No: 003771) mouse strains were obtained from Jackson Laboratory. The L3mbt3 deletion mutant (MBT-1-/+, B6;129-L3mbt|3tm1Tmiy) mouse strain was previously described ${ }^{41}$. All animal experiments including breeding, housing, genotyping, and sample collection were conducted in accordance with the animal protocols approved by the institutional Animal Use and Care Committee (IACUC) and complied with all relevant ethical regulations at University of Nevada, Las Vegas. All procedures were conducted according to the National Institutes of Health (NIH) 
Guide for Care and Use of Laboratory Animals. The UNLV IACUC is an AAALAC approved facility and meets the NIH Guide for the Care and Use of Animals. For embryos analyses, usually 3 pairs of the $\operatorname{LBMBTL3}(-/+)$ male and female mice (10-12 weeks old) in three cages, each with 1 male and 1 female, were bred in the late afternoon and the breeding plugs were examined in the female mice in next morning. The positive plugs were count as embryonic day 1 (E1) and the pregnant female mice between E14-E17.5 were euthanized by the primary method of $\mathrm{CO}_{2}$ asphyxiation, followed by cervical dislocation (secondary method), as approved by the institutional IACUC committee. Usually a single pregnant female mouse produced about 6-8 embryos, which segregated at the Mendelian ratio, usually with 1-2 L3MBTL3 $(-/-), 1-2$ wildtype and 3-4 heterozygous $\angle 3 M B T L 3(-/+)$ embryos. The $\angle 3 M B T L 3$ null embryos between E17.5-19.5 usually died and became disintegrated so they were excluded from protein analyses. For the analysis of $\mathrm{mSWI} / \mathrm{SNF}$ proteins in LSD ${ }^{\mathrm{fl} / \mathrm{fl}} / \mathrm{Nestin}$-Cre mice. Usually 3-4 pairs of the LSD $1^{\text {flox/flox }}$ male and LSD $1^{\text {flox/+ }} /$ Nestin-Cre mice female mice (10-12 weeks old) were bred. The animals were collected immediate after birth to avoid any delay in sample analysis. The brains of the mice were dissected for protein or immunostaining analysis. For immunostaining, embryos or dissected brains were fixed in $4 \%$ paraformaldehyde (PFA) at $4^{\circ} \mathrm{C}$ overnight and embedded in Optimal cutting temperature compound (O.C.T) according to standard procedures ${ }^{27}$. Sections (10 $\mu \mathrm{m}$ thick, coronal) were stained with specific antibodies and counter-stained with 4',6-diamidino-2-phenylindole (DAPI). Images were acquired with the Nikon A1Rsi Confocal LSM. The sample size was chosen on the basis of our experience on L3MBTL3 or Lsd1 mutant mice and on cultured cells in order to detect the mSWI/SNF proteins for differences of at least $50 \%$ between the wildtype and mutant groups ${ }^{41}$. In the experimental analyses for examination of proteins, the investigators were unaware of the genotypes of the experimental embryos. The investigators also randomly analyzed the wildtype, heterozygous and homozygous knockdown embryos.

\section{Analysis of proteins and DNA from embryos}

The experimental procedures for embryo isolation were approved by the UNLV Institutional Animal Use and Care Committee (IACUC). The $L 3 m b t / 3$ embryos from the euthanized pregnant female mice or dissected brains from the $L s d 1$ mice, washed with PBS, and lysed in the NP40 lysis buffer ${ }^{41}$. For the The nuclear and cytosolic fractions were separated by centrifugation. Genomic DNA was isolated from nuclear pellets by Zymo genomic DNA-tissue prep kit and quantified. Proteins in the cytosolic suppernant of the lysates were quantified by protein assay dye (Bio-Rad), equalized, and boiled for 15 minutes after addition of $1 \%$ SDS and $5 \%$ beta-mercaptoethanol to the lysates. Proteins were resolved in protein gel and analyzed by Western blotting.

\section{Statistical information}


Experiments were usually performed with at least three independent repeats (biological replicates) to ensure the results. For animal experiments, triplicated breeding was used to obtain statistically significant number of embryos; and statistically significant differences between means of protein levels in the control wildtype and knockout mutants were compared using two-tailed equal-variance independent Student's t-test ${ }^{41}$. All other data were determined using a two-tailed equal-variance independent Student's t-test. The data in all figures met the assumption of normal distribution for tests. Different data sets were considered to be statistically significant when the P-value was $<0.05\left(^{\star}\right), 0.01$ (**) or $\left.0.001{ }^{(\star \star *}\right)^{55}$.

\section{Data Availability}

The authors declare that the data supporting the findings of this study are available within the paper and its supplementary information files. The data that support the findings of this study are also available from the corresponding author upon reasonable request.

\section{Declarations}

\section{Acknowledgements}

This work was supported by grants from National Institutes of Health (R15CA254827 to HS and R15GM131255 and R01 GM140185 to HZ).

\section{Author Contributions}

H. Zhang directed the study; P. Guo designed and performed most of the experiments; N. Hoang, J Sanchez, K. E. H. Zhang, Rajawasam, and K. Trinidad assisted some experiments. H. Sun interpret mouse embryonic stem cell data, provided intellectual contribution and helpful discussions. P. Guo and $\mathrm{H}$. Zhang wrote the manuscript.

\section{Competing interests}

The authors declare no competing interests.

\section{References}

1 Ho, L. \& Crabtree, G. R. Chromatin remodelling during development. Nature 463, 474-484, doi:10.1038/nature08911 (2010). 
2 Kadoch, C. et al. Proteomic and bioinformatic analysis of mammalian SWI/SNF complexes identifies extensive roles in human malignancy. Nat Genet 45, 592-601, doi:10.1038/ng.2628 (2013).

3 Kadoch, C. \& Crabtree, G. R. Mammalian SWI/SNF chromatin remodeling complexes and cancer: Mechanistic insights gained from human genomics. Sci Adv 1, e1500447, doi:10.1126/sciadv.1500447 (2015).

4 Ho, L. et al. An embryonic stem cell chromatin remodeling complex, esBAF, is an essential component of the core pluripotency transcriptional network. Proc Natl Acad Sci U S A 106, 5187-5191, doi:10.1073/pnas.0812888106 (2009).

$5 \mathrm{Ho}$, L. et al. An embryonic stem cell chromatin remodeling complex, esBAF, is essential for embryonic stem cell self-renewal and pluripotency. Proc Natl Acad Sci U S A 106, 5181-5186, doi:10.1073/pnas.0812889106 (2009).

6 Yan, Z. et al. BAF250B-associated SWI/SNF chromatin-remodeling complex is required to maintain undifferentiated mouse embryonic stem cells. Stem Cells 26, 1155-1165, doi:10.1634/stemcells.20070846 (2008).

7 Singhal, N., Esch, D., Stehling, M. \& Scholer, H. R. BRG1 Is Required to Maintain Pluripotency of Murine Embryonic Stem Cells. Biores Open Access 3, 1-8, doi:10.1089/biores.2013.0047 (2014).

8 Singhal, N. et al. Chromatin-Remodeling Components of the BAF Complex Facilitate Reprogramming. Cell 141, 943-955, doi:10.1016/j.cell.2010.04.037 (2010).

9 Han, D. et al. SRG3, a core component of mouse SWI/SNF complex, is essential for extra-embryonic vascular development. Dev Bio/315, 136-146, doi:10.1016/j.ydbio.2007.12.024 (2008).

10 Bultman, S. et al. A Brg1 null mutation in the mouse reveals functional differences among mammalian SWI/SNF complexes. Mol Cel/ 6, 1287-1295, doi:10.1016/s1097-2765(00)00127-1 (2000).

11 Tuoc, T. C. et al. Chromatin regulation by BAF170 controls cerebral cortical size and thickness. Dev Cell 25, 256-269, doi:10.1016/j.devcel.2013.04.005 (2013).

12 Wang, X. et al. Oncogenesis caused by loss of the SNF5 tumor suppressor is dependent on activity of BRG1, the ATPase of the SWI/SNF chromatin remodeling complex. Cancer Res 69, 8094-8101, doi:10.1158/0008-5472.CAN-09-0733 (2009).

13 Helming, K. C. et al. ARID1B is a specific vulnerability in ARID1A-mutant cancers. Nat Med 20, 251254, doi:10.1038/nm.3480 (2014).

14 Schick, S. et al. Systematic characterization of BAF mutations provides insights into intracomplex synthetic lethalities in human cancers. Nat Genet 51, 1399-1410, doi:10.1038/s41588-019-0477-9 (2019). 
15 Narayanan, R. et al. Loss of BAF (mSWI/SNF) Complexes Causes Global Transcriptional and Chromatin State Changes in Forebrain Development. Cell Rep 13, 1842-1854, doi:10.1016/j.celrep.2015.10.046 (2015).

16 Wilson, B. G. et al. Residual complexes containing SMARCA2 (BRM) underlie the oncogenic drive of SMARCA4 (BRG1) mutation. Mol Cell Bio/ 34, 1136-1144, doi:10.1128/MCB.01372-13 (2014).

17 Helming, K. C., Wang, X. \& Roberts, C. W. M. Vulnerabilities of mutant SWI/SNF complexes in cancer. Cancer Cell 26, 309-317, doi:10.1016/j.ccr.2014.07.018 (2014).

18 Greer, E. L. \& Shi, Y. Histone methylation: a dynamic mark in health, disease and inheritance. Nat Rev Genet 13, 343-357, doi:10.1038/nrg3173 (2012).

19 Margueron, R. \& Reinberg, D. Chromatin structure and the inheritance of epigenetic information. Nat Rev Genet 11, 285-296, doi:10.1038/nrg2752 (2010).

20 Shi, Y. et al. Histone demethylation mediated by the nuclear amine oxidase homolog LSD1. Cel/ 119, 941-953, doi:10.1016/j.cell.2004.12.012 (2004).

21 Wang, J. et al. Opposing LSD1 complexes function in developmental gene activation and repression programmes. Nature 446, 882-887, doi:10.1038/nature05671 (2007).

22 Wang, J. et al. The lysine demethylase LSD1 (KDM1) is required for maintenance of global DNA methylation. Nat Genet 41, 125-129, doi:10.1038/ng.268 (2009).

23 Adamo, A. et al. LSD1 regulates the balance between self-renewal and differentiation in human embryonic stem cells. Nat Cell Bio/ 13, 652-659, doi:10.1038/ncb2246 (2011).

24 Saleque, S., Kim, J., Rooke, H. M. \& Orkin, S. H. Epigenetic regulation of hematopoietic differentiation by Gfi-1 and Gfi-1b is mediated by the cofactors CoREST and LSD1. Mol Cell 27, 562-572, doi:10.1016/j.molcel.2007.06.039 (2007).

25 Zhang, C. et al. LSD1 demethylase and the methyl-binding protein PHF20L1 prevent SET7 methyltransferase-dependent proteolysis of the stem-cell protein SOX2. J Biol Chem 293, 3663-3674, doi:10.1074/jbc.RA117.000342 (2018).

26 Zhang, C. et al. Proteolysis of methylated SOX2 protein is regulated by L3MBTL3 and CRL4(DCAF5) ubiquitin ligase. J Biol Chem 294, 476-489, doi:10.1074/jbc.RA118.005336 (2019).

27 Christopher, M. A. et al. LSD1 protects against hippocampal and cortical neurodegeneration. Nat Commun 8, 805, doi:10.1038/s41467-017-00922-9 (2017).

28 Zhang, X., Wen, H. \& Shi, X. Lysine methylation: beyond histones. Acta Biochim Biophys Sin (Shanghai) 44, 14-27, doi:10.1093/abbs/gmr100 (2012). 
29 Abbas, E. et al. Conditional Loss of BAF (mSWI/SNF) Scaffolding Subunits Affects Specification and Proliferation of Oligodendrocyte Precursors in Developing Mouse Forebrain. Front Cell Dev Bio/ 9, 619538, doi:10.3389/fcell.2021.619538 (2021).

$30 \mathrm{Kim}, \mathrm{S}$. K. et al. SET7/9 methylation of the pluripotency factor LIN28A is a nucleolar localization mechanism that blocks let-7 biogenesis in human ESCs. Cell Stem Cel/ 15, 735-749, doi:10.1016/j.stem.2014.10.016 (2014).

$31 \mathrm{Fu}$, L. et al. Set7 mediated Gli3 methylation plays a positive role in the activation of Sonic Hedgehog pathway in mammals. Elife 5, doi:10.7554/eLife.15690 (2016).

32 Lee, J. Y. et al. LSD1 demethylates HIF1alpha to inhibit hydroxylation and ubiquitin-mediated degradation in tumor angiogenesis. Oncogene 36, 5512-5521, doi:10.1038/onc.2017.158 (2017).

33 Nishioka, K. et al. Set9, a novel histone H3 methyltransferase that facilitates transcription by precluding histone tail modifications required for heterochromatin formation. Genes Dev 16, 479-489, doi:10.1101/gad.967202 (2002).

34 Wang, H. et al. Purification and functional characterization of a histone H3-lysine 4-specific methyltransferase. Mol Cel/ 8, 1207-1217, doi:10.1016/s1097-2765(01)00405-1 (2001).

35 Fang, L. et al. A methylation-phosphorylation switch determines Sox2 stability and function in ESC maintenance or differentiation. Mol Cel/ 55, 537-551, doi:10.1016/j.molcel.2014.06.018 (2014).

36 Calnan, D. R. et al. Methylation by Set9 modulates Fox03 stability and transcriptional activity. Aging (Albany NY) 4, 462-479, doi:10.18632/aging.100471 (2012).

37 Kontaki, H. \& Talianidis, I. Lysine methylation regulates E2F1-induced cell death. Mol Cel/ 39, 152-160, doi:10.1016/j.molcel.2010.06.006 (2010).

38 Yang, J. et al. Reversible methylation of promoter-bound STAT3 by histone-modifying enzymes. Proc Natl Acad Sci U S A 107, 21499-21504, doi:10.1073/pnas.1016147107 (2010).

39 Yang, X. D. et al. Negative regulation of NF-kappaB action by Set9-mediated lysine methylation of the RelA subunit. EMBO J 28, 1055-1066, doi:10.1038/emboj.2009.55 (2009).

40 Esteve, P. O. et al. Regulation of DNMT1 stability through SET7-mediated lysine methylation in mammalian cells. Proc Natl Acad Sci U S A 106, 5076-5081, doi:10.1073/pnas.0810362106 (2009).

41 Leng, F. et al. Methylated DNMT1 and E2F1 are targeted for proteolysis by L3MBTL3 and CRL4(DCAF5) ubiquitin ligase. Nat Commun 9, 1641, doi:10.1038/s41467-018-04019-9 (2018).

42 Tronche, F. et al. Disruption of the glucocorticoid receptor gene in the nervous system results in reduced anxiety. Nat Genet 23, 99-103, doi:10.1038/12703 (1999). 
43 Mashtalir, N. et al. Modular Organization and Assembly of SWI/SNF Family Chromatin Remodeling Complexes. Cell 175, 1272-1288 e1220, doi:10.1016/j.cell.2018.09.032 (2018).

44 Chen, J. \& Archer, T. K. Regulating SWI/SNF subunit levels via protein-protein interactions and proteasomal degradation: BAF155 and BAF170 limit expression of BAF57. Mol Cell Bio/ 25, 9016-9027, doi:10.1128/MCB.25.20.9016-9027.2005 (2005).

45 Sohn, D. H. et al. SRG3 interacts directly with the major components of the SWI/SNF chromatin remodeling complex and protects them from proteasomal degradation. J Biol Chem 282, 10614-10624, doi:10.1074/jbc.M610563200 (2007).

46 Hayashi, S. \& McMahon, A. P. Efficient recombination in diverse tissues by a tamoxifen-inducible form of Cre: a tool for temporally regulated gene activation/inactivation in the mouse. Dev Bio/ 244, 305-318, doi:10.1006/dbio.2002.0597 (2002).

47 Bonasio, R., Lecona, E. \& Reinberg, D. MBT domain proteins in development and disease. Semin Cell Dev Bio/ 21, 221-230, doi:10.1016/j.semcdb.2009.09.010 (2010).

48 Wei, D. et al. SNF5/INI1 deficiency redefines chromatin remodeling complex composition during tumor development. Mol Cancer Res 12, 1574-1585, doi:10.1158/1541-7786.MCR-14-0005 (2014).

49 Petroski, M. D. Mechanism-based neddylation inhibitor. Chem Bio/ 17, 6-8, doi:10.1016/j.chembiol.2010.01.002 (2010).

50 Northcott, P. A. et al. Multiple recurrent genetic events converge on control of histone lysine methylation in medulloblastoma. Nat Genet 41, 465-472, doi:10.1038/ng.336 (2009).

51 Kar, S. P. et al. Genome-Wide Meta-Analyses of Breast, Ovarian, and Prostate Cancer Association Studies Identify Multiple New Susceptibility Loci Shared by at Least Two Cancer Types. Cancer discovery 6, 1052-1067, doi:10.1158/2159-8290.CD-15-1227 (2016).

52 Andlauer, T. F. et al. Novel multiple sclerosis susceptibility loci implicated in epigenetic regulation. Science advances 2, e1501678, doi:10.1126/sciadv.1501678 (2016).

53 Lotta, L. A. et al. Integrative genomic analysis implicates limited peripheral adipose storage capacity in the pathogenesis of human insulin resistance. Nat Genet 49, 17-26, doi:10.1038/ng.3714 (2017).

54 Arai, S. \& Miyazaki, T. Impaired maturation of myeloid progenitors in mice lacking novel Polycomb group protein MBT-1. EMBO J 24, 1863-1873, doi:10.1038/sj.emboj.7600654 (2005).

55 Fay, D. S. \& Gerow, K. A biologist's guide to statistical thinking and analysis. WormBook : the online review of C. elegans biology, 1-54, doi:10.1895/wormbook.1.159.1 (2013).

\section{Figures}


a.

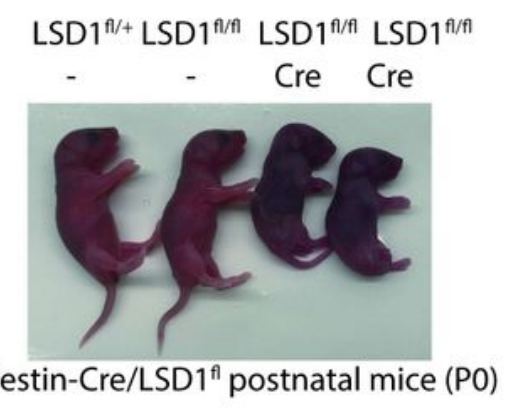

c.

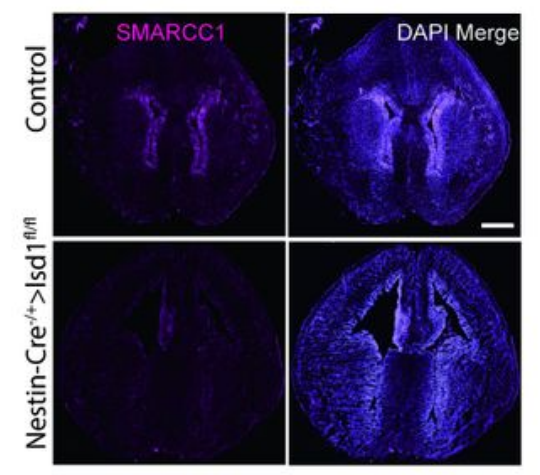

e.

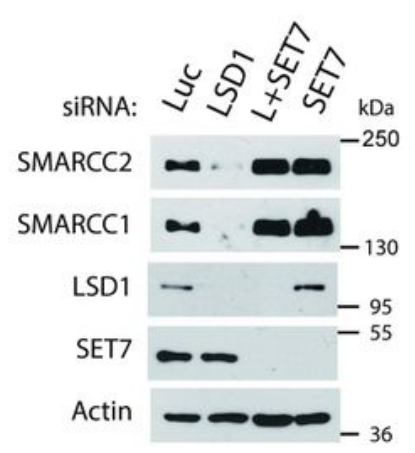

b.

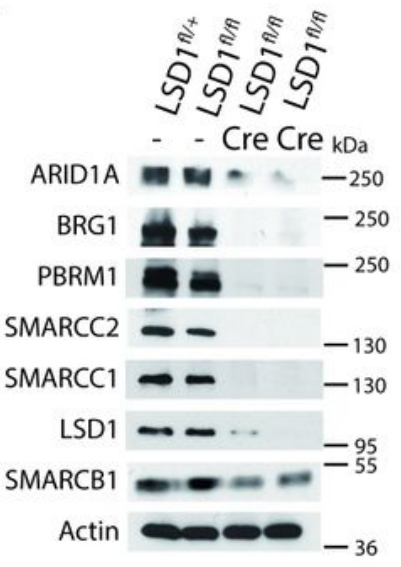

Nestin-Cre/LSD $1^{\text {f }}$ Embryos (P0)

d.

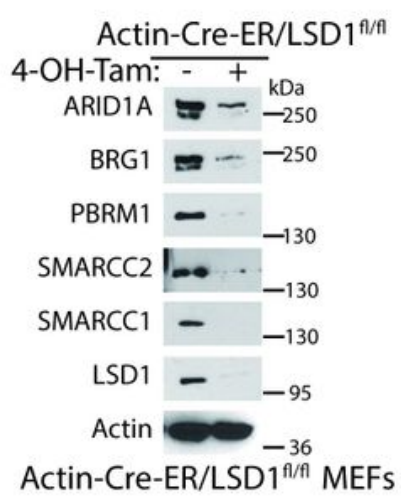

f.

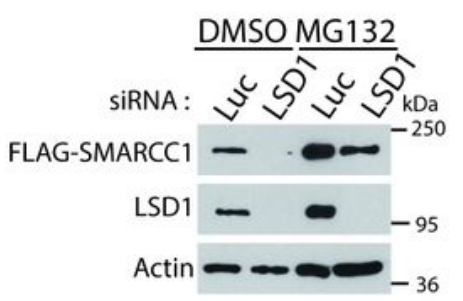

Figure 1

\section{Figure 1}

Dramatic loss of mSWI/SNF proteins in LSD1 null mice.

a. Nestin-Cre directed conditional inactivation of mouse $L s d 1$ gene causes immediate postnatal death after birth (P0). b. Homozygous loss of $L s d 1$ leads to dramatic reduction of mSWI/SNF proteins. Brain extracts from LSD1 ${ }^{\text {flox/+}}$, LSD $1^{\text {flox/flox }}$, and LSD1 ${ }^{\text {flox/flox} / N e s t i n-C r e ~ m i c e ~ w e r e ~ W e s t e r n ~ b l o t t e d ~ t o ~ a n a l y z e ~}$ 
indicated SWI/SNF proteins. c. Reduction of SMARCC1 in LSD1 1 fl/fl/Nestin-Cre mice. Brain cryo-sections

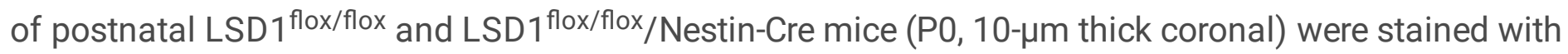
anti-SMARCC1 antibody, counter-stained with 4',6-diamidino-2-phenylindole (DAPI). Scale bar: $500 \mu \mathrm{m}$. d. Excision of $L s d 1$ by $4-\mathrm{OH}-\mathrm{TAM}$ in $L s d 7^{f / f} /$-actin-CreER reduces $\mathrm{mSWI} / \mathrm{SNF}$ proteins in MEFs. Embryonic fibroblasts from CAGGCre-ER ${ }^{T M} / L S D 1^{\text {fl/fl }}$ mouse embryos (E13.5) were treated with 4-hydroxytamoxifen ( $20 \mathrm{mg} / \mathrm{ml}$ ) for 12 hours to delete $L s d 1$ by inducible Actin-Cre-ER to analyze SWI/SNF proteins. e. SET7 silencing stabilizes SMARCC1 and SMARCC2. HeLa cells were transfected with $50 \mathrm{nM}$ siRNAs of luciferase (Luc), LSD1, LSD1+SET7, or SET7 for 48 hours and indicated proteins were analyzed by Western blotting. Actin serves a loading control. f. LSD1 silencing-induced Flag-SMARCC1 downregulation is sensitive to MG132. H1299 cells stably expressing Flag-tagged SMARCC1 under the retroviral LTR promoter control were transfected with $50 \mathrm{nM}$ of luciferase and LSD1 siRNAs for 48 hours and then treated with either dimethylsulfoxide (DMSO) or $5 \mu \mathrm{g} / \mathrm{ml} \mathrm{MG132}$ for last 6 hours before lysing the cells for Western blotting of Flag-SMARCC1, LSD1, and actin. 
a.

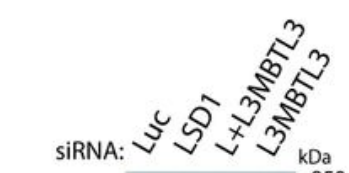

SMARCC1 $-{ }^{-250}$

LSD1 $\sim-\boldsymbol{m}^{-130}$

L3MBTL3 - - - 95

Actin - - - - -36

c.

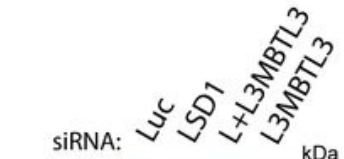

ARID1A $\mathbb{E}=\boldsymbol{\omega}=1.1,-250$

BRG1 - - - ${ }^{-250}$

BRM - - - - -250

PBRM1 - $\boldsymbol{\sim}^{-250}$

LSD1 - $\boldsymbol{- 1 3 0}^{-130}$

L3MBTL3 - - - 95

Actin - - - -36

e.

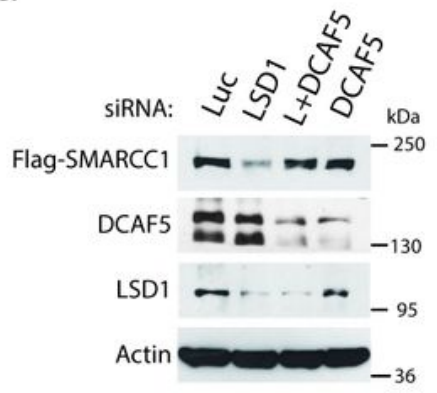

IP: $\lg \alpha \alpha-$ L3MBTL3

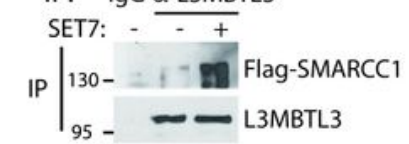

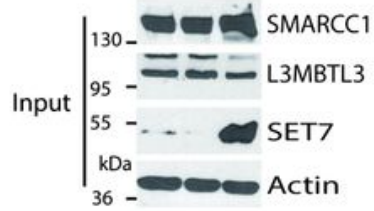

b.

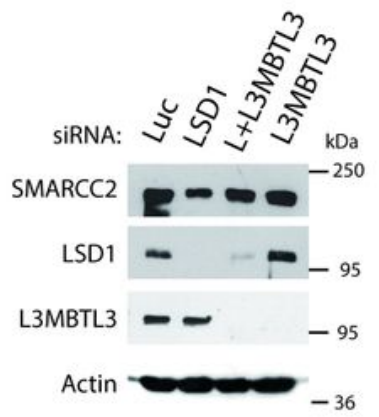

d.

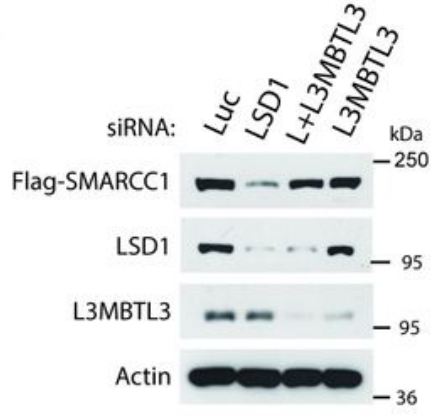

f.

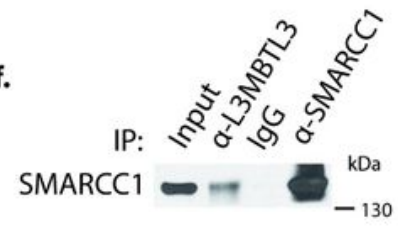

L3MBTL3 - - - -95

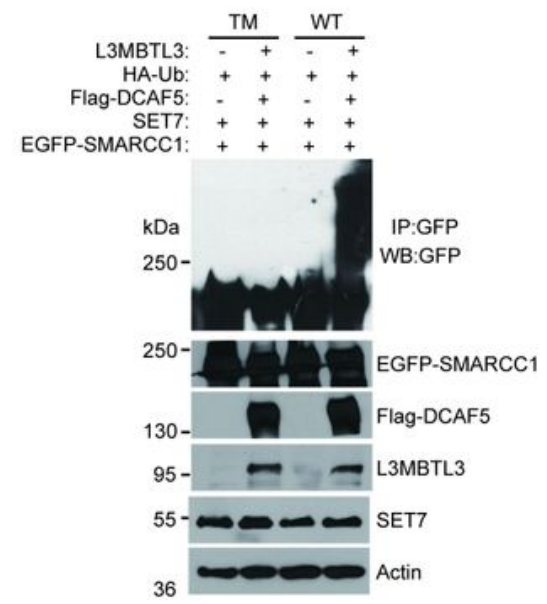

Figure 2

Figure 2

\section{Regulation of SMARCC1 and SMARCC2 by L3MBTL3 and DCAF5.}

a-c. L3MBTL3 silencing stabilizes endogenous mSWI/SNF proteins in HeLa cells. HeLa cells were transfected with $50 \mathrm{nM}$ siRNAs of luciferase, LSD1, LSD1+L3MBTL3, or L3MBTL3 for 48 hours. The levels of SMARCC1 (a), SMARCC2 (b) and other indicated mSWI/SNF proteins were analyzed by Western 
blotting. d. H1299 cells stably expressing Flag-SMARCC1 were transfected with $50 \mathrm{nM}$ of luciferase, LSD1, LSD1+L3MBTL3, and L3MBTL3 siRNAs for 48 hours and Flag-SMARCC1 levels were analyzed by Western blotting. e. DCAF5 silencing stabilizes Flag-SMARCC1. H1299 cells expressing Flag-SMARCC1 were transfected with $50 \mathrm{nM}$ of luciferase, LSD1, LSD1+DCAF5, and DCAF5 siRNAs and analyzed as 2d. f. Endogenous L3MBTL3 interacts with SMARCC1. L3MBTL3 and SMARCC1 were immunoprecipitated from 293 cells and blotted with respective antibodies. g. SET7 stimulates L3MBTL3-SMARCC1 interaction. H1299 cells expressing Flag-SMARCC1 were transfected with either control empty vector or SET7 expressing vector for 48 hours and cell lysates were immunoprecipitated by lgG control or antiL3MBTL3 antibody and indicated proteins were detected by antibodies against indicated proteins. $\mathbf{h}$. EGFP-tagged wildtype and triple mutant of SMARCC1 expressing constructs were co-transfected into 293 cells together with vectors expressing HA-tagged ubiquitin (HA-Ub), and SET7 in the presence or absence of L3MBTL3 and DCAF5 expressing constructs as indicated. Proteins were immunoprecipitated with antiGFP antibodies and Western blotted with the anti-GFP-SMARCC1 and other antibodies against indicated proteins. 
a.
H3-K4:
MARTK * QTARKSTGGKAPRKQLA
Sox2-K42:
NOKNSPDRVK * RPMNAFMVWSRGQRRKM
Sox2-K117:
YKYRPRRKTK * TLMKKDKYTLPGGLLAP
DNMT1-K142:
SKPRTPRRSK * SDGEAKRSRDPPASASQ
E2F1-K185:
GIQLIAKKSK *NHIQWLGSHTTVGVGGR
SMARCC1-K201:
QGTFTDEKSK *ASHHIYPYSSSQDDEEW
SMARCC1-K482:
EFFNGKNKSK * TPEIYLAYRNFMIDTYR
SMARCC1-K615:
YSKKTLAKSK * GASAGREWTEQETLLLL
SMARCC2-K382:
GPSTPYTKSK * RGHREEEQEDLTKDMDE
EFFNGKNKSK * TPEIYLAYRNFMIDTYR
SMARCC2-K475:
TKKNVPSKSK *AAASATREWTEQETLLL

b.

unmethylated K482 peptide: RALPEFFNGKNKSK(me0)TPEIYLAYRNFMIDTC methylated $\mathbf{K 4 8 2}$ peptide: RALPEFFNGKNKSK(me1)TPEIYLAYRNFMIDTC unmethylated K615 peptide: GLRTDIYSKKTLAKSK(me0)GASAGREWTEQETC methylated K615 peptide: GLRTDIYSKKTLAKSK(me1)GASAGREWTEQETC

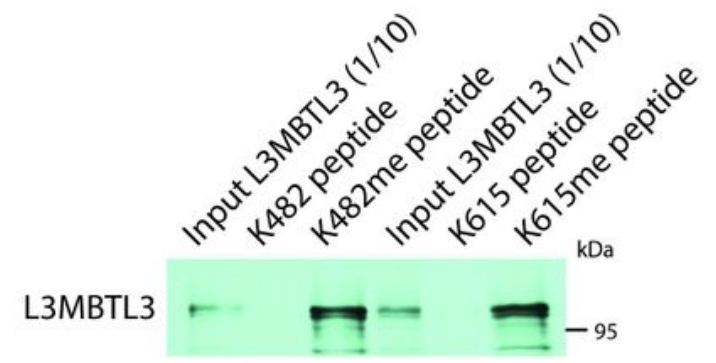

c.

anti-K482me antibody

GST GST-LSD1

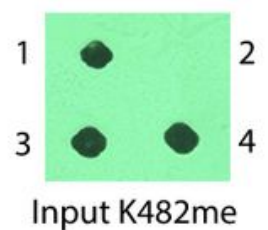

d.

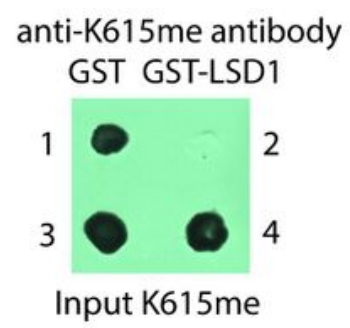

Figure 3

Figure 3

\section{L3MBTL3 and LSD1 recognize the methylated K482 and K615 in SMARCC1.}

a. The consensus lysine residues $\left(K^{*}\right)$ methylated by SET7 in H3K4-like methylation motifs with the R/K$\mathrm{S} / \mathrm{T} / \mathrm{V}-\mathrm{K}^{*}$ consensus sequences. b. L3MBTL3 preferentially binds to the monomethylated K482 and K615 peptides. Sulfolink-agarose immobilized SMARCC1 peptides containing the monomethylated K482 and 
$\mathrm{K} 615$, or cognate unmethylated peptides were incubated with $20 \mathrm{ml}$ of in vitro translated and 35Smethionine labeled L3MBTL3 for 1 hour at room temperature. L3MBTL3 binding to the peptide resins was analyzed by autoradiography after protein electrophoresis separation. c. and d. LSD1 demethylates methylated K482 and K615 in SMARCC1. Purified $1 \mathrm{mg}$ of GST (spot 1) or GST-LSD1 proteins (spot 2) were incubated with $100 \mathrm{ng}$ of mono-methylated K482 (c) or mono-methylated K615 (d) peptides for 4 hours at $37^{\circ} \mathrm{C}$ and the resulting peptides and control input methylated peptides (spots 3 and 4) were spotted onto nitrocellulose membrane. The methylated peptides were detected by affinity purified antimono-methylated K482 (c) or K615 (d) peptide antibodies (see Supplementary Fig 1a and 1b), respectively, as indicated. 
a.

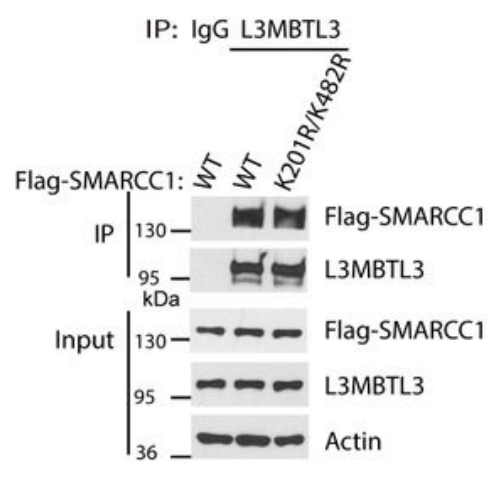

c.

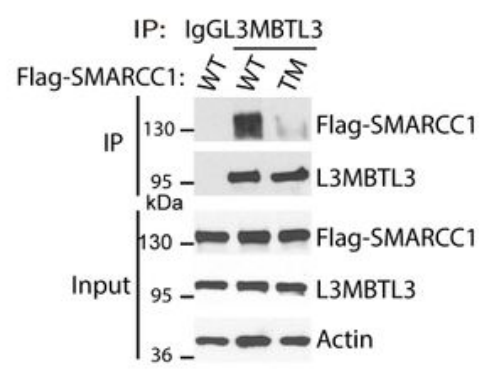

e.

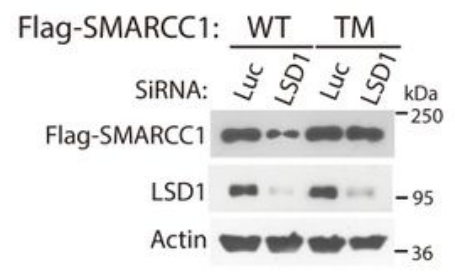

g.

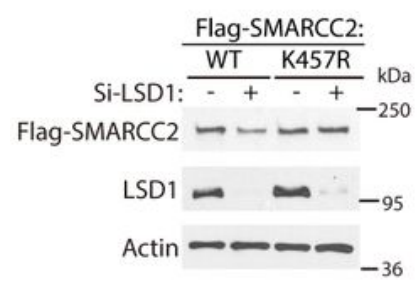

b.

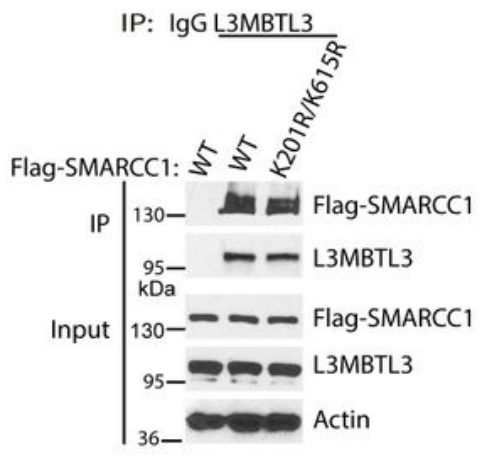

d.

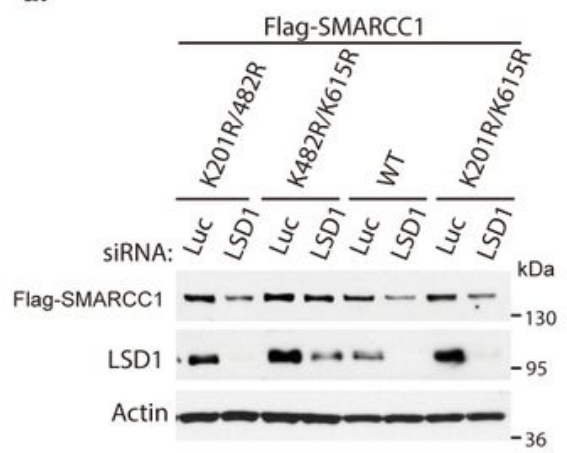

f.

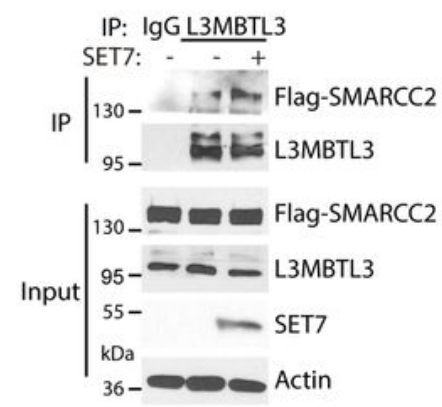

h.

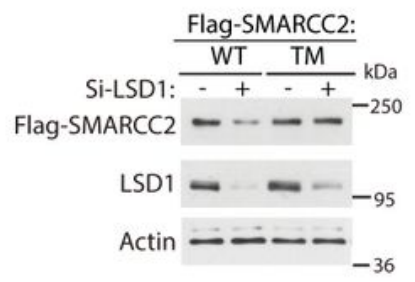

Figure 4

Figure 4

\section{L3MBTL3 interacts with the methylated K482 and K615 to regulate SMARCC1.}

a-c. K482 and K615 of SMARCC1 are required for interacting with L3MBTL3. The wildtype, K201R/K482R (a) and K201R/K615R (b) double mutants, or K201R/K482R/K615R (c) triple mutants of Flag-SMARCC1 were transfected into 293 cells for 48 hours and cell lysates were immunoprecipitated by anti-L3MBTL3 
antibodies or IgG (control). The interactions between Flag-SMARCC1 and its mutants were detected by antibodies against indicated proteins. $\boldsymbol{d}$ and $\mathbf{e}$. Mutations of K482 and K615 confer resistance to LSD1 silencing. H1299 cells stably expressing the wildtype, K201R/K482R, K482/K615R, or K201R/K615R double mutants (d), or the K201R/K482R/K615R triple mutant of SMARCC1 (e) were transfected with 50 nM siRNAs of luciferase or LSD1 for 48 hours and the levels of Flag-SMARCC1 protein and its mutants were analyzed by Western blotting. f. SET7 promotes interaction between L3MBTL3 and SMARCC2. H1299 cells stably expressing Flag-SMARCC2 were transfected with either the control empty vector or SET7 expressing vector for 48 hours and cell lysates were immunoprecipitated by lgG control or antiL3MBTL3 antibodies. The interaction between SMARCC2 and L3MBTL3 were analyzed. $\mathbf{g}$. and $\mathbf{h}$. The K475R and K382R/K475R/K592R mutants of SMARRC2 are resistant to LSD1 silencing. H1299 cells expressing the wildtype, K475R single (g) or K382R/K475R/K592R (h) triple mutant of SMARCC2 were transfected with $50 \mathrm{nM}$ siRNAs of luciferase or LSD1 for 48 hours and the levels of Flag-SMARCC2 wildtype and mutants were analyzed by Western blotting as indicated. 
a.

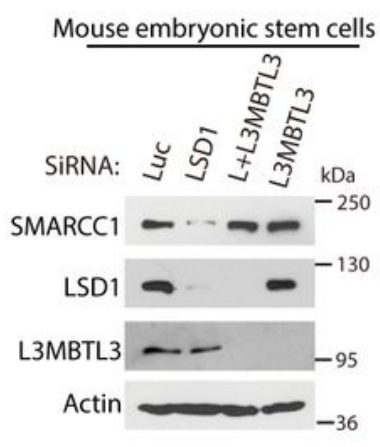

c.

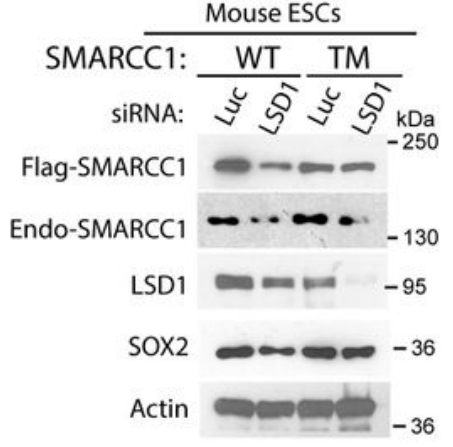

f.

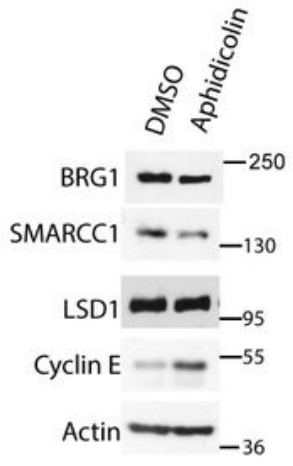

b.

Mouse embryonic stem cells

Flag-SMARCC1 Flag-SMARCC1-TM

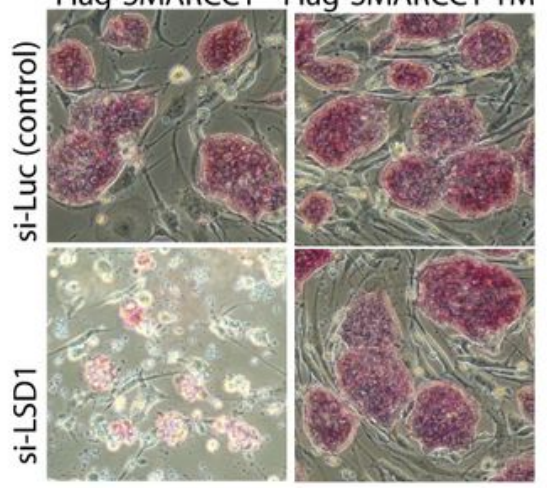

d.

e.

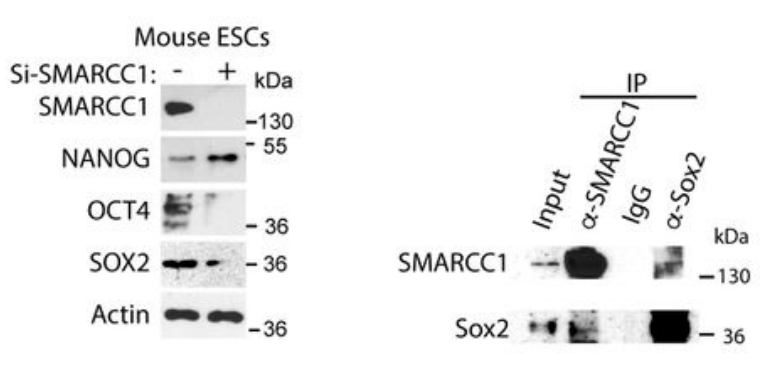

Figure 5

Figure 5

SMARCC1 is a critical target of LSD1 in mouse ESCs.

a. L3MBTL3 silencing stabilizes SMARCC1 in mouse embryonic stem cells. The mESCs were transfected with $50 \mathrm{nM}$ of luciferase, LSD1, LSD1+L3MBTL3, and L3MBTL3 siRNAs for 48 hours. The levels of SMARCC1, LSD1, L3MBTL3, and actin were examined by Western blotting. b. and c. SMARCC1 triple 
mutant confers resistance to LSD1 silencing. The mESCs stably expressing the wildtype and the K201R/K482R/K615R triple mutant of Flag-SMARCC1 were transfected with $50 \mathrm{nM}$ siRNAs of luciferase or LSD1for 40 hours, stained with alkaline phosphatase (AP), and cell images were acquired (b) with Nikon ECLIPSE Ti-S microscope equipped with NIS-Elements BR 3.1 software. The levels of endogenous and ectopically expressed SMARCC1, LSD1, SOX2, and actin were examined by Western blotting with respective antibodies (c). d. SMARCC1 silencing downregulates OCT4 and SOX2 and upregulates NANOG in mESCs. mESCs were transfected with $50 \mathrm{nM}$ of luciferase (-) and SMARCC1 (+) siRNAs for 40 hours. SMARCC1, OCT4, SOX2, NANOG, and actin were examined by Western blotting. e. Endogenous SOX2 and SMARCC1 proteins were immunoprecipitated from mESCs and reciprocally blotted with respective antibodies. Input: 1/10 of cell lysates were used. $\mathrm{f}$. Aphidicolin causes downregulation of BRG1 and SMARCC 1 in mESCs. The mESCs were treated with dimethyl sulfoxide (DMSO) or $5 \mathrm{mg} / \mathrm{ml}$ aphidicolin for 12 hours and the levels of BRG1, SMARCC1, LSD1, cyclin E were examined by Western blotting. $\mathbf{g}$. L3MBT3 silencing stabilizes BRG1 and SMARCC1 in aphidicolin treated mESCs. mESCs were transfected with $50 \mathrm{nM}$ of luciferase or L3MBTL3 siRNAs for 20 hours and then treated with dimethyl sulfoxide (DMSO) or $5 \mathrm{mg} / \mathrm{ml}$ aphidicolin for another 12 hours. BRG1, SMARCC1, LSD1, L3MBTL3, and SOX2 were examined by Western blotting. 
a.

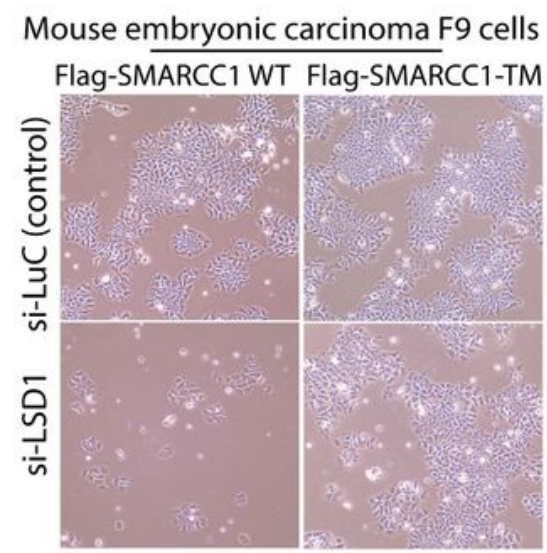

b.

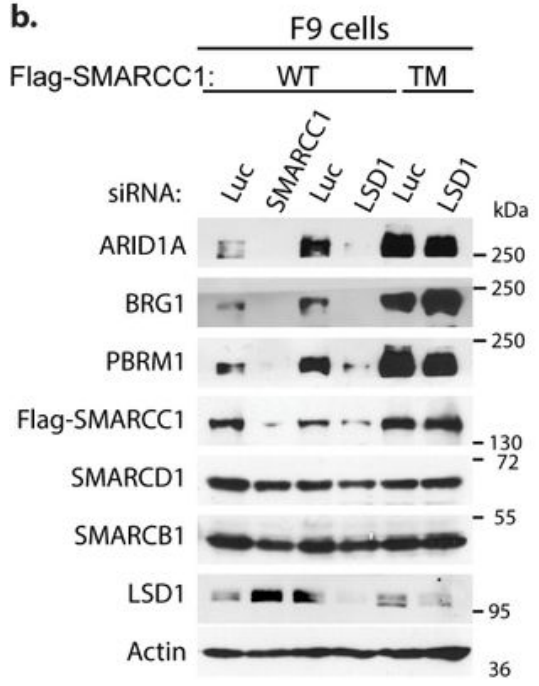

d.

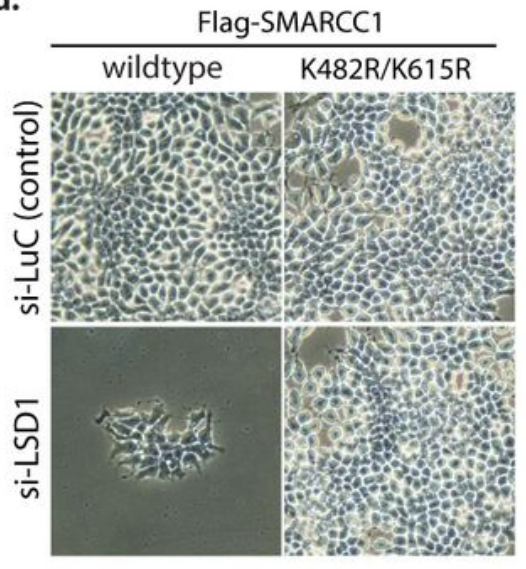

c.

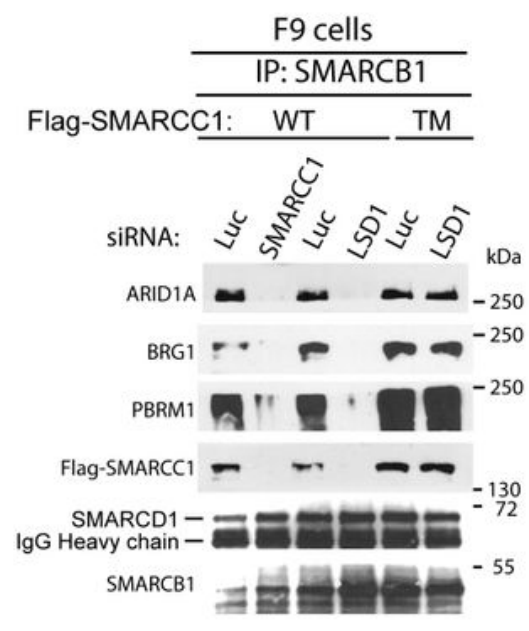

e.

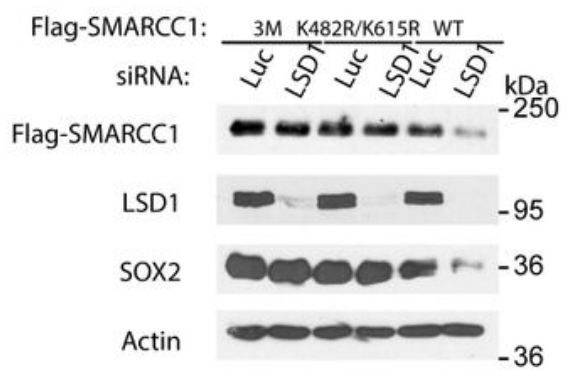

Figure 6

Figure 6

Mutations of SMARCC1 confer the resistance to LSD1 silencing in F9 embryonic carcinoma cells.

a. The wildtype and the K201R/K482R/K615R triple mutant of Flag-SMARCC1 were stably expressed in mouse F9 embryonic carcinoma cells. The cells were transfected with with $50 \mathrm{nM}$ siRNAs of luciferase or LSD1 for 40 hours and cell images were acquired (a) and mSWI/SNF proteins were examined by Western 
blotting with respective antibodies (Supplementary Fig. 1d). b. and c. F9 cells stably expressing the wildtype and the K201R/K482R/K615R triple mutant of Flag-SMARCC1 were transfected with $50 \mathrm{nM}$ of luciferase, SMARCC1, and LSD1 siRNAs for 40 hours. One set of lysates was used for direct Western blotting (b) and the other set for immunoprecipitation with anti-SMARCB1 antibodies and then Western blotting with antibodies for indicated SWI/SNF proteins (c). d. F9 cells stably expressing the wildtype and K482R/K615R double mutant of SMARCC1 were transfected with $50 \mathrm{nM}$ of luciferase and LSD1 siRNAs for 40 hours and cell images were acquired. e. The level of SOX2 is restored by SMARCC1 mutants in F9 cells. F9 cells stably expressing the wildtype, the K482R/K615R double mutant, and the K201R/K482R/K615R triple mutant of Flag-SMARCC1 were transfected with $50 \mathrm{nM}$ of luciferase and LSD1 siRNAs for 40 hours. The levels of Flag-SMARCC1, SOX2, LSD1, and actin were examined by Western blotting. 
a.

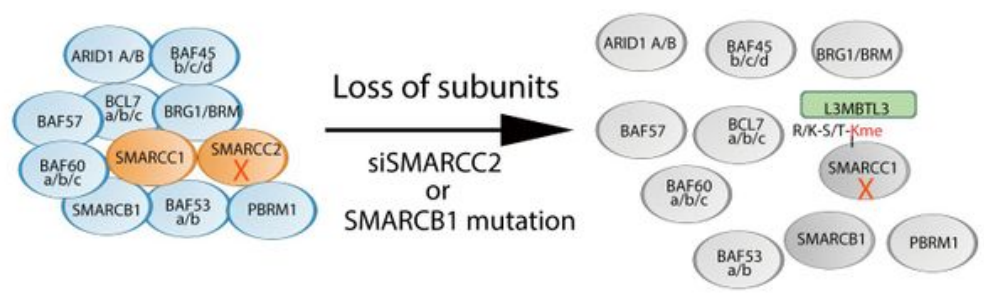

b.

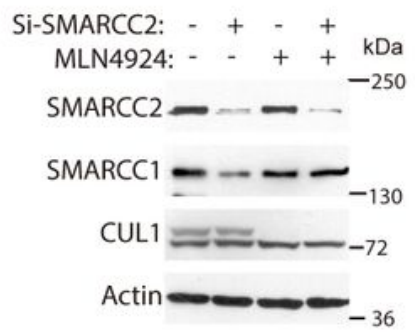

d.

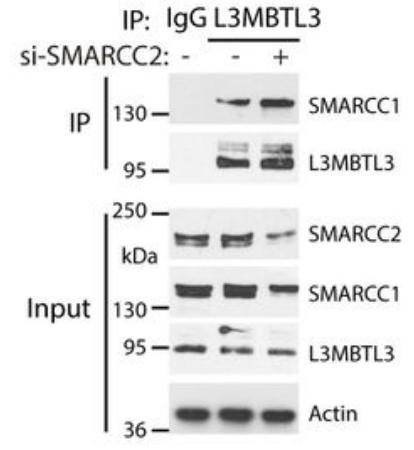

c.

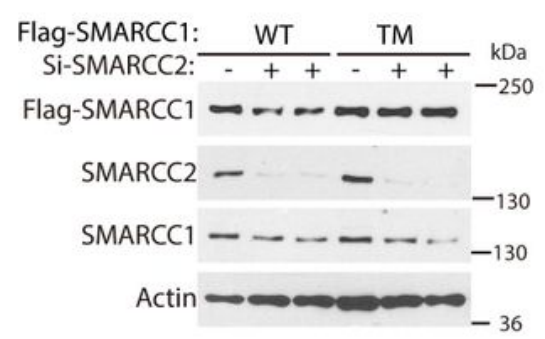

e.

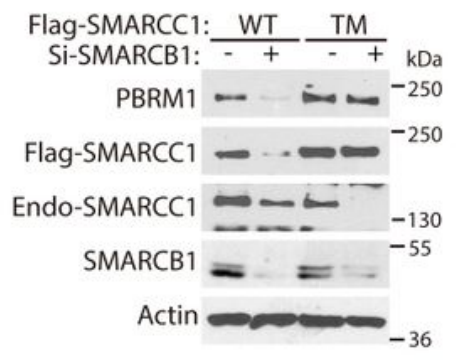

f.

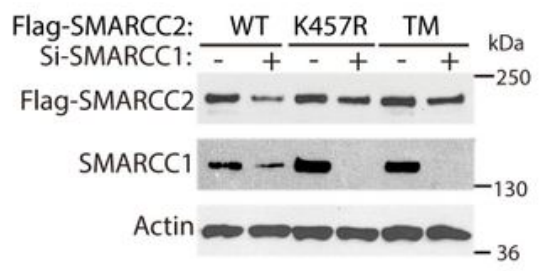

Figure 7

Figure 7

L3MBTL3 regulates the proteolysis of SMARCC1 and SMARCC2 during the disassembly of the SWI/SNF complexes.

a. Either loss or mutation of SWI/SNF components such as SMARCC2 or SMARCB1 leads to the proteolytic degradation of other subunits including SMARCC1. b. The degradation of SMARCC1 induced 
by SMARCC2 is re-stabilized by MLN4924. HeLa cells were transfected with $50 \mathrm{nM}$ siRNAs of luciferase or SMARCC2 for 42 hours and the cells were treated with or without $2 \mu \mathrm{M}$ MLN4924, a neddylation inhibitor, for 6 hours as indicated. SMARCC1, SMARCC2, and CUL1 proteins were analyzed by Western blotting. c. SMARCC1 triple mutant is resistant to SMARCC2 silencing. H1299 cells stably expressing wildtype or the K201R/K482R/K615R triple mutant of SMARCC1 were transfected with $50 \mathrm{nM}$ siRNAs of luciferase or SMARCC2 for 48 hours and the levels of Flag-SMARCC1 and its mutant and endogenous SMARCC1 and SMARCC2 were analyzed by Western blotting. $\mathbf{d}$. Loss of SMARCC2 enhances the interaction between L3MBTL3 and SMARCC1. HeLa cells were transfected with $50 \mathrm{nM}$ siRNAs of luciferase or SMARCC2 for 48 hours and the interaction between SMARCC1 and L3MBTL3 was examined by immunoprecipitation and Western blotting. e. SMARCC1 triple mutant confers the resistance of to SMARCB1 silencing. H1299 cells expressing wildtype or the K201R/K482R/K615R triple mutant of FlagSMARCC1 were transfected with $50 \mathrm{nM}$ siRNAs of luciferase or SMARCB1 siRNAs for 48 hours and FlagSMARCC, the triple mutant, and PBRM1 proteins were examined by Western blotting. $\mathbf{f}$. The SMARCC2 K475R single and K382R/K475R/K592R triple mutants are resistant to SMARCC1 silencing. H1299 cells expressing wildtype or the K382R/K475R/K592R triple mutant of SMARCC2 were transfected with $50 \mathrm{nM}$ siRNAs of luciferase or SMARCC1 siRNAs for 48 hours and Flag-SMARCC2 and its mutant proteins were examined. 
a.

L3MBTL3 embryos (E17.5)

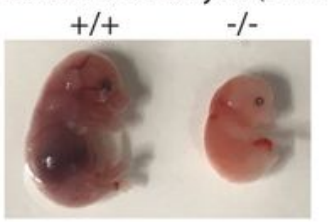

c. L3MBTL3 embryos $\frac{\mathrm{E} 9.5}{-/-+/+-/-+/+-/-+/+\mathrm{kDa}}$

SMARCC1 $\mathbf{0} \boldsymbol{- \infty}-250$

Actin

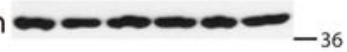

b.

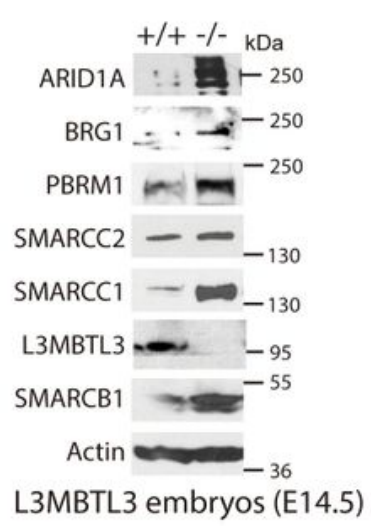

d.

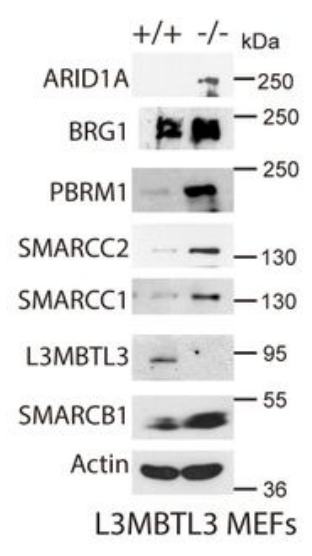

e.

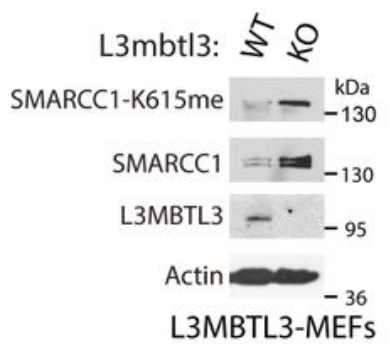

f.

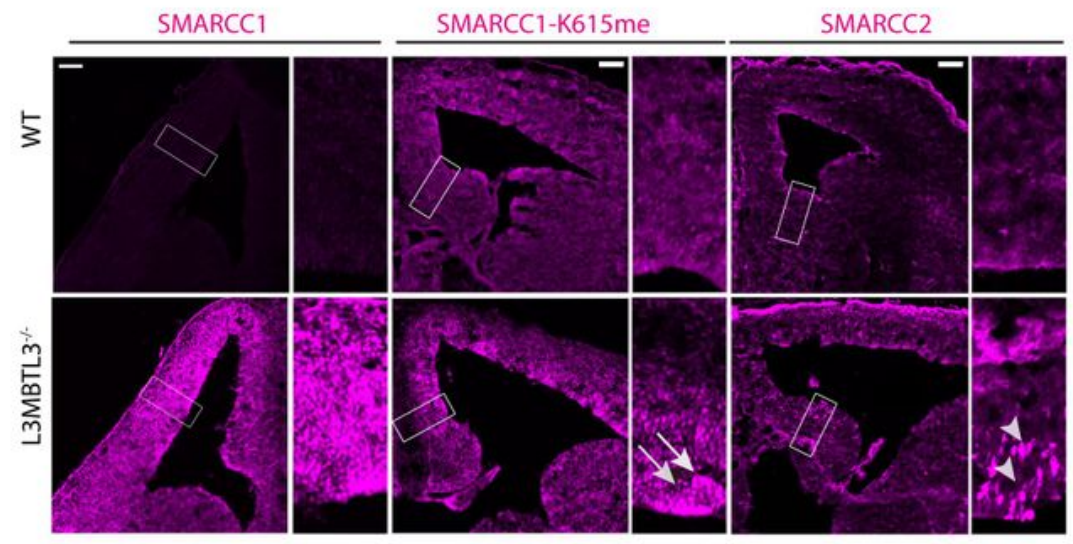

g.

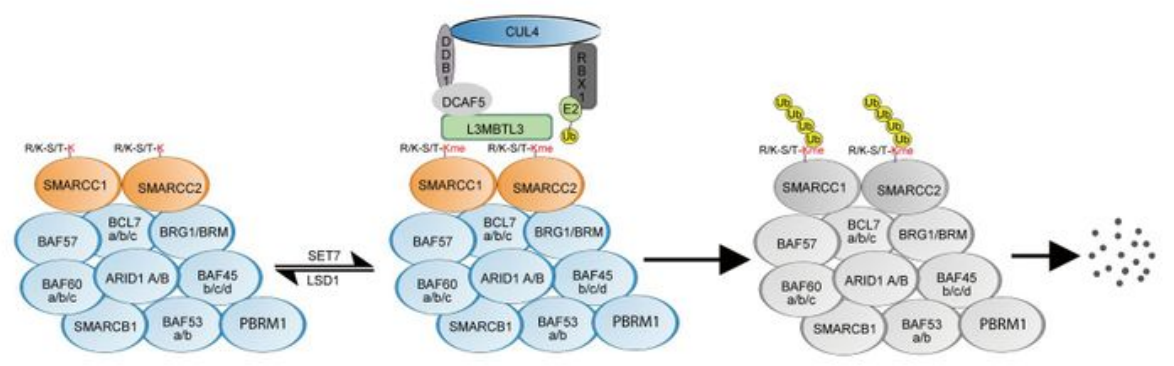

Figure 8

Figure 8

L3MBTL3 regulates the proteolysis of SMARCC1 and SMARCC2 during the mouse development.

a. Homozygous deletion of mouse $L 3 m b t / 3(-/-)$ is embryonic lethal between E15.5 17.5. b. $L 3 m b t / 3$ null mutation causes the accumulation of $\mathrm{mSWI} / \mathrm{SNF}$ proteins. Total lysates from the heads of mouse L3mbt3 (+/+) and deletion (-/-) mutant embryos (equal total proteins) were analyzed by Western blotting 
with antibodies for the indicated mSWI/SNF proteins. c. Accumulation of SMARCC1 in L3mbt/3 null embryos at various embryonic dates. Lysates from the heads of wildtype and $L 3 m b t / 3$ deletion embryos were collected at various embryonic dates (E9.5, E14.5, and E17.5) and analyzed for SMARCC1 protein levels. d. L3MBTL3 (-/-) MEFs accumulate mSWI/SNF proteins. Mouse embryonic fibroblasts from the wildtype and $\angle 3 m b t / 3$ deletion mutant embryos (E13.5) were examined for indicated SWI/SNF proteins by Western blotting. e. The K615 mono-methylated SMARCC1 protein accumulates in L3mbt3 deletion (-/-) MEFs. The $L 3 m b t 3(+/+)$ and deletion (-/-) mutant MEFs were analyzed for mono-methylated $\mathrm{K} 615$ of SMARCC1, total SMARCC1, and L3MBTL3 proteins by Western blotting. f. SMARCC1, mono-methylated K615 form of SMARCC1, and SMARCC2 accumulate in mouse $L 3 \mathrm{mbt} / 3$ (-/-) brain sections. Embryonic brain cryo-sections of wildtype and $\angle 3 m b t / 3$ deletion mutant (E14.5, 10- $\mu \mathrm{m}$ thick coronal) were immunostained with anti-SMARCC1, mono-methylated K615 of SMARCC1, and SMARCC2 antibodies. Boxed regions are enlarged and arrowheads or arrows indicate the increased SMARCC1-K615me or SMARCC2 regions in the brains. Scale bar: $100 \mu \mathrm{m}$. g. Model: Specific lysine residues of SMARCC1 and SMARCC2, core components of the SWI/SNF complex, are methylated by SET7 methyltransferase and the levels of methyl-lysines are reversibly maintained by LSD1 demethylase. L3MBTL3 preferentially binds to the methyl-lysine residues of SMARCC1 or SMARCC2 to recruit the CRL4 ${ }^{\text {DCAF5 }}$ ubiquitin E3 ligase complex to target the methylated proteins for ubiquitin-dependent proteolysis, promoting the disassembly and proteolytic degradation of the mSWI/SNF complex.

\section{Supplementary Files}

This is a list of supplementary files associated with this preprint. Click to download.

- SupplementaryFigure1.tif

- SupplementaryFigure2.tif 